\title{
Composition of Wide Bandgap Semiconductor Materials and Nanostructures Measured by Atom Probe Tomography and Its Dependence on the Surface Electric Field
}

Lorenzo Mancini, ${ }^{\dagger}$ Nooshin Amirifar, ${ }^{\dagger}$ Deodatta Shinde, ${ }^{\dagger}$ Ivan Blum, ${ }^{\dagger}$ Matthieu Gilbert, ${ }^{\dagger}$ Angela Vella, ${ }^{\dagger}$ François Vurpillot, ${ }^{\dagger}$ Williams Lefebvre, ${ }^{\dagger}$ Rodrigue Lardé, ${ }^{\dagger}$ Etienne Talbot, ${ }^{\dagger}$ Philippe Pareige, ${ }^{\dagger}$ Xavier Portier, ${ }^{\ddagger}$ Ahmed Ziani, ${ }^{\ddagger}$ Christian Davesnne, ${ }^{\ddagger}$ Christophe Durand, ${ }^{\S}$ Joël Eymery, ${ }^{\S}$ Raphaël Butté," Jean-François Carlin," Nicolas Grandjean," and Lorenzo Rigutti*, ${ }^{\dagger}$

† Groupe de Physique des Matériaux, UMR 6634 CNRS, University and INSA of Rouen, Normandie University, 76800 St. Etienne du Rouvray, France

${ }^{\ddagger}$ CIMAP/CEA, UMR CNRS 6252/ENSICAEN/Université de Caen Basse Normandie, 6, Boulevard Maréchal Juin, 14050 Caen, France

${ }^{\S}$ CEA/CNRS/Université Joseph Fourier, CEA, INAC, SP2M, 17 rue des Martyrs, 38054 Grenoble Cedex 9, France

"Institute of Condensed Matter Physics (ICMP), Ecole Polytechnique Fédérale de Lausanne (EPFL), CH-1015 Lausanne, Switzerland

\begin{abstract}
Atom probe tomography allows for threedimensional reconstruction of the elemental distribution in materials at the nanoscale. However, the measurement of the chemical composition of compound semiconductors may exhibit strong biases depending on the experimental parameters used. This article reports on a systematic analysis of the composition measurement of III-N binary (AIN, GaN) and ternary compounds ( $\mathrm{InGaN}$, InAlN), $\mathrm{MgO}$, and $\mathrm{ZnO}$ by laserassisted tomographic atom probe as a function of laser power and applied DC bias. We performed separate series of measurements at constant bias, constant laser pulse energy,
\end{abstract} and constant detection rate and a spatial analysis of the surface

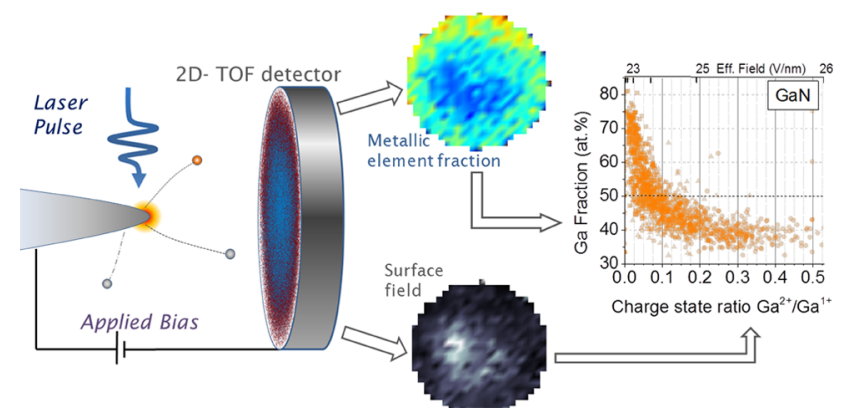

field through detector hitmap ratios of elemental charge states. As a result, (i) we can determine the separate roles of laser energy and surface field- the latter being the dominant factor under standard conditions of analysis; (ii) we compare the behavior of different samples and (iii) different materials; and (iv) we critically discuss the reliability of the measurement of InxGa1- $\mathrm{xN}$ and InxAl1- $\mathrm{xN}$ alloy fractions and of the $\mathrm{Tb}$ concentration in rare-earth-doped $\mathrm{ZnO}$.

\section{INTRODUCTION}

The elemental composition of materials can be in principle measured with $3 \mathrm{D}$ subnanometer resolution by laser-assisted atom probe tomography (LA-APT). ${ }^{1}$ For this reason, this technique is currently being more and more applied in different domains of nanoscale science and technology, such as metallurgy, ${ }^{2}$ electronics, ${ }^{3-11}$ and geosciences. ${ }^{12}$ However, the measurement of composition is accurate only if all atomic species present in the analyzed specimen are field evaporated and detected with the same rate: unfortunately, this hypothesis does not generally hold in the case of nonmetallic compounds. Due to different physical mechanisms, compound semiconductors and dielectrics exhibit a complex field evaporation behavior, translating into phenomena such as the detection of molecular ions and ion clusters in the mass spectra ${ }^{13-15}$ or the dissociation of molecular ions after evaporation. ${ }^{16}$ These complex mechanisms, which are not fully understood yet, can also lead to an erroneous measurement of the elemental composition of the sample.

Earlier work on one-dimensional pulsed-voltage and pulsedlaser atom probe already showed that a correct composition in III-V semiconductors is not automatically obtained but may be obtained by properly tuning the experimental parameters. ${ }^{17-19}$ In ref 19, for instance, strong compositional biases are found in the laser-assisted atom probe measurements of GaAs, and an interpretation of them is proposed.

With the development of 3D APT assisted by femtosecond lasers, the problem of measuring the correct composition has gained a further degree of complexity. First, the experimentalist has to face it for an increasingly larger choice of materials. 
Table 1. Selected Properties of the Binary Compound Semiconductors Analyzed by Atom Probe Tomography

\begin{tabular}{|c|c|c|c|c|}
\hline material & crystal structure & bandgap @ $300 \mathrm{~K}(\mathrm{eV})$ & binding energy (eV/pair) & fractional ionic character \\
\hline $\mathrm{GaN}$ & hexagonal wurtzite & $3.43^{\mathrm{bl}}$ & 10.2 (th.) -11.0 (th. $)^{d, e}$ & $0.51^{a}$ \\
\hline AlN & hexagonal wurtzite & $6.13^{\mathrm{b} 1}$ & 11.4 (th.) -13.3 (th. $)^{d, e}$ & $0.72^{a}$ \\
\hline $\mathrm{ZnO}$ & hexagonal wurtzite & $3.3^{b}$ & 7.2 (th.) -7.52 (exp. $)^{c}$ & $0.53^{a}-0.61$ \\
\hline $\mathrm{MgO}$ & cubic halite & $7.2^{f}-7.8^{g}$ & $10.17($ th. $)-10.26(\exp )^{c}$ & $0.84^{h}$ \\
\hline
\end{tabular}

Table 2. List of Analyzed Samples and Their Selected Properties ${ }^{a}$

\begin{tabular}{|c|c|c|c|c|}
\hline material/system & sample type & method of growth or synthesis & \# of tips analyzed & crystal orientation of APT tip axis \\
\hline $\mathrm{GaN}$ & microwire & $\mathrm{MOCVD}^{b}$ & $2(1 \mathrm{FP})$ & {$[0001] c$-axis } \\
\hline AlN & thin film on sapphire & $\mathrm{MBE}^{c}$ & $2(\mathrm{FP})$ & {$[0001] c$-axis } \\
\hline $\mathrm{InGaN} / \mathrm{GaN}$ & multiple quantum wells in microwire & $\operatorname{MOCVD}^{d}$ & 1 & {$[1-100] m$-axis } \\
\hline $\mathrm{GaN} / \mathrm{InAlN}$ & multiple quantum wells in microwire & $\operatorname{MOCVD}^{e}$ & 1 & {$[1-100] m$-axis } \\
\hline $\mathrm{ZnO}$ & nanowire & $\operatorname{MOCVD}^{f}$ & $1(\mathrm{FP})$ & {$[0001] c$-axis } \\
\hline $\mathrm{ZnO}$ & polycrystalline bulk & SPS of $\mathrm{ZnO}$ powder ${ }^{g}$ & 1 & undefined \\
\hline $\mathrm{ZnO}: \mathrm{Tb}$ & polycrystalline thin film ( $1 \mu \mathrm{m}$ thickness) & $\mathrm{RFMS}^{h}$ & $1(\mathrm{FP})$ & [0001] (preferential) \\
\hline $\mathrm{MgO}$ & bulk & Arc melting $^{i}$ & $2(\mathrm{FP})$ & {$[001]$} \\
\hline
\end{tabular}

${ }^{a}$ Definition of the acronyms concerning the synthesis: MBE, molecular beam epitaxy; MOCVD, metalorganic chemical vapor deposition; RFMS, radio-frequency magnetron sputtering; SPS, spark plasma sintering. The acronym FP indicates that the sample has been analyzed applying the full protocol described in section $2.7 .{ }^{b} \operatorname{Ref} 37 .{ }^{c} \operatorname{Ref} 38 .{ }^{d} \operatorname{Ref} 39 .{ }^{e} \operatorname{Ref} 40 . f_{\operatorname{Ref}} 41 .{ }^{g} \operatorname{Ref} 42 .{ }^{h} \operatorname{Ref} 43 .{ }^{i} \operatorname{Ref} 44$.

Second, it becomes clear that not only the measured global composition is affected by the experimental parameters but also the locally measured composition may be biased. Indeed, even in homogeneous samples local biases are due to the inhomogeneous geometric or crystallographic properties of the tip surface ${ }^{20}$ or to the asymmetric distribution of the laser pulse. $^{21,22}$ Some recent works have analyzed this problem in binary compounds such as $\mathrm{GaSb}^{21} \mathrm{GaN}^{20,22-24}$ and $\mathrm{MgO},{ }^{25}$ and more in-depth analyses appeared in order to discuss the reliability of the measurements of alloy fraction in the ternary compound InGaN, a system of technological impact due to its application to light-emitting diodes. ${ }^{24}$ For other wide gap materials already analyzed using an atom probe, such as $\mathrm{ZnO}^{15}$ and related oxides ${ }^{26,27}$ or $\mathrm{AlN}^{28}$ the problem of the correct composition measurement has not been systematically addressed yet.

The main goal of this work is to provide a systematic analysis of compositional biases in wide bandgap compound semiconductors, adopting a well-defined methodology on selected materials of interest in the domain of nanoscale electronics. The developed methods allow for direct comparison with future measurements performed in any atom probe instrument and are virtually portable to other materials. In particular, we study the compositional biases measured in LA-APT for a set of four binary wide bandgap compounds, $\mathrm{GaN}, \mathrm{AlN}, \mathrm{ZnO}$, and $\mathrm{MgO}$, providing systematic and comparable data. By applying a protocol consisting of separate series of measurements at constant detection rate (flux), at constant laser energy, and at constant applied bias, we find that the fraction of metallic and nonmetallic elements in these materials is nearly independent of the incident laser energy-at least in standard conditions for reliable atom probe analysis. This allows us to represent these biases as a function of a surface field indicator that we choose as the charge state ratio $\mathrm{Me}^{2+} / \mathrm{Me}^{1+}$, where $\mathrm{Me}^{n+}$ is the amount of atoms of the metallic element measured as an $n$-times charged ion in the mass spectrum. In this way, it becomes possible not only to quantitatively compare different samples of the same material but also to qualitatively compare the behavior of different materials.
Furthermore, we study the ternary InGaN and InAlN nitride alloys, and $\mathrm{ZnO}: \mathrm{Tb}$, in order to verify the conditions under which the measurements of alloy fractions and doping density are reliable. The analysis of InGaN- and InAlN-based multiple quantum wells - systems for which the reconstructed volume is a priori known-allows us to establish the global amount of atoms lost either by diffusion and neutral evaporation or by preferential evaporation.

Finally, we attempt a global interpretation of the different evaporation behaviors on the basis of the set of results and of the available literature considering the problem from a theoretical point of view. We also indicate which experimental and theoretical aspects of field ion evaporation could be worth investigating in order to gain further insight into the physical mechanisms leading to the nonstoichiometric detection of species in the atom probe.

\section{EXPERIMENTAL SECTION}

2.1. Analyzed Materials and Samples. The role of the experimental parameters on the composition determination of binary compound semiconductors was performed on four different systems: two III-nitride semiconductors, GaN and AlN, and two metal oxides, $\mathrm{ZnO}$ and $\mathrm{MgO}$. This choice was suggested by the possibility of comparing the behavior of materials issued by the same families of compounds but having different properties. Some of those properties are listed in Table 1. All materials are wide bandgap dielectrics but with significant differences in their electronic structure, translating into different values of bandgap, binding energy per pair, and fractional ionic character. It is worth noticing that the listed properties refer to bulk materials and thus will not be sufficient to robustly explain the observed field ion evaporation behavior, as the latter is a pure surface phenomenon. However, these properties could serve as a starting point for the development of a more detailed theoretical analysis and as a guide for the investigation of other III-V and II-VI materials.

The analyzed samples (including those containing ternary compounds) are listed in Table 2 . The reported properties refer to the sample type and its method of synthesis, along with the 
references providing further detail on their structural and chemical properties. The number of analyzed tips and the crystal orientation of the tip axis during the APT analysis are also reported.

2.2. Sample Preparation. $\mathrm{MgO}, \mathrm{AlN}$, and $\mathrm{ZnO}$ bulk and thin film materials have been prepared by standard focused ion beam (FIB)-assisted lift out of a region of interest of the material to analyze, followed by soldering on a tungsten tip by $\mathrm{Pt}-\mathrm{C}$ ion beam assisted deposition and subsequent annular milling. All FIB milling steps have been performed with $30 \mathrm{keV}$ $\mathrm{Ga}^{2+}$ ions, except for the final cleaning step, performed with 2 $\mathrm{keV} \mathrm{Ga}^{2+}$ ions. GaN, InGaN/GaN, and InAlN/GaN microwire samples have been either collected by micromanipulation under optical microscope and glued by conductive epoxy on tungsten tips (in this case the axis of analysis is the crystal $c$-axis) or picked up by FIB-assisted micromanipulation in a scanning electron microscope (SEM), soldered by $\mathrm{Pt}-\mathrm{C}$ on $\mathrm{W}$ tips, and subsequently annularly milled by FIB, as described in ref 45 . $\mathrm{ZnO}$ nanowire samples have been collected by optical micromanipulation under an optical microscope and subsequently soldered on $\mathrm{W}$ tips by electron beam-assisted $\mathrm{Pt}-\mathrm{C}$ or $\mathrm{W}-\mathrm{C}$ deposition. No annular milling was needed due to their low apex radius.

2.3. Atom Probe: Principles and Instrumentation. The schematic representation of a laser-assisted atom probe experiment is reported in Figure 1. The sample is a field-

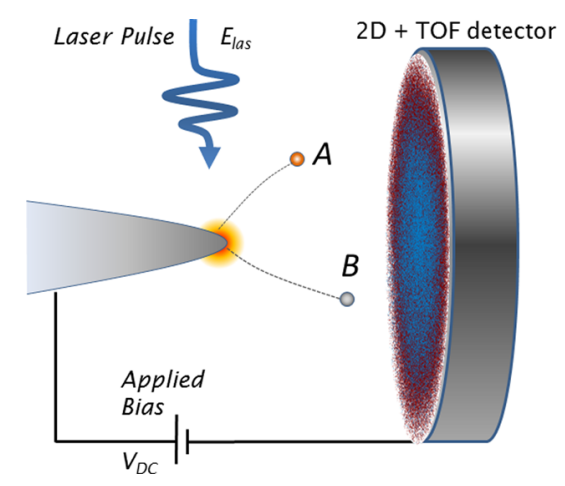

Figure 1. Schematic representation of an atom probe experiment.

emission tip set at temperature $T \leq 80 \mathrm{~K}$, with an apex radius lower than $\sim 100 \mathrm{~nm}$. This tip is polarized with a positive applied DC bias $V_{\mathrm{DC}}$, yielding an intense field at the tip apex. Field ion evaporation is triggered by a femtosecond laser pulse with energy $E_{\text {las }}$. Surface atoms may ionize and leave the surface, flying toward a detector sensitive to the impact position of the ion and to its time-of-flight. The detection rate $\Phi$, also called flux, is defined as the number of detected ions per pulse. The time-of-flight of the ions allows for the identification of their mass/charge ratio, i.e., - with some limitations - of their chemical identity. Mass/charge histograms, or mass spectra, allow thus for the measurement of chemical composition. Furthermore, the impact position on the detector makes it possible to reconstruct the original position of each ion on the tip through a back-projection algorithm. It becomes thus possible to retrieve multidimensional information, such as $2 \mathrm{D}$ surface maps of emitted species or the $3 \mathrm{D}$ reconstruction of the chemical composition of a given volume.

All samples studied in this work, with the exception of a polycrystalline $\mathrm{ZnO}$ tip, were analyzed in a Cameca laserassisted wide-angle tomographic atom probe (LAWATAP) with a flight length of around $10 \mathrm{~cm}$ and a multichannel plate/ advanced delay line detector (MCP/ADLD) ${ }^{46}$ with $8 \mathrm{~cm}$ diameter, corresponding to a field of view of $15^{\circ}$. The temperature was controlled by a closed-loop He cryostat and set for each material to a typical value for standard analysis. The tip samples were studied under UV illumination with $400 \mathrm{fs}$ laser pulses at the wavelength of $343 \mathrm{~nm}$ and at the repetition frequency of $100 \mathrm{kHz}$, with a laser spot diameter of $\sim 60 \mu \mathrm{m}$ diameter (the pulse energy $E_{\text {las }}=1 \mathrm{~nJ}$ corresponds thus roughly to an average pulse intensity $I_{\text {las }}=0.22 \mathrm{~W} \mu \mathrm{m}^{-2}$ ). The polycrystalline $\mathrm{ZnO}$ tip was analyzed at $T=80 \mathrm{~K}$ in a Cameca laser-assisted FlexTAP, in a configuration having a flight length of $80 \mathrm{~cm}$ and a field of view of $30^{\circ}$.

2.4. Mass Spectra of Binary Compound Semiconductors. The first step for the measurement of the composition of an atom probe specimen is a correct identification of the peaks appearing in the mass spectrum. Figure 2 reports the typical mass spectra measured by atom probe for each of the four analyzed binary compounds. Each mass spectrum contains around $10^{5}$ identified ions. In the following, we will briefly review the main features of the mass spectra of each material.

2.4.1. GaN. The mass spectrum of $\mathrm{GaN}$ is reported in Figure 2(a). All elemental and molecular peaks can be identified unambiguously, with the exception of the peak at $14 \mathrm{amu}$. As the experimental conditions do not allow for a quantification of the ${ }^{15} \mathrm{~N}$ isotope, this peak could be attributed to $\mathrm{N}_{2}{ }^{2+}, \mathrm{N}^{1+}$, or a mixture of both. In our data, there are no elements for discriminating between the different cases. Unambiguous results would be possible if the material was enriched in ${ }^{15} \mathrm{~N}$, but this is not the case for our samples. However, we hypothesize that the peak is related to $\mathrm{N}_{2}{ }^{2+}$, which has a lifetime of the order of seconds, sufficiently long to be detected. ${ }^{47}$ In any case it should be noticed that the attribution of this peak to $\mathrm{N}^{1+}$ would slightly change the measured composition but would not influence the overall dependence of the measured composition on the experimental parameters, as discussed in section 2.6. No measurable trace of $\mathrm{Ga}^{3+}$ was found within the explored interval of experimental parameters.

2.4.2. AIN. Figure 2(b) reports a typical mass spectrum of AlN. With respect to GaN, similar considerations apply to the peak at $14 \mathrm{amu}$. It is interesting to notice that in AlN it is possible to measure a significant amount of $\mathrm{N}^{2+}$, which never occurred in GaN mass spectra measured in this work. This suggests that evaporation takes place at a higher surface field than in $\mathrm{GaN}$. The presence of $\mathrm{Al}^{3+}$ is interesting as it marks a deviation from the Kingham charge state statistics of fieldevaporated atoms. ${ }^{48}$ The attribution of the peak at $14 \mathrm{amu}$ to $\mathrm{N}^{1+}$ would decrease the $\mathrm{N}$ fraction by a value roughly equal to $5 \%$ throughout the explored range of parameters.

2.4.3. $\mathrm{ZnO}$. The mass spectrum of a nominally undoped $\mathrm{ZnO}$ nanowire is reported in Figure 2(c). The attribution of peaks in $\mathrm{ZnO}$ should take into account two facts: (i) the peak at 32 amu should be attributed to two different contributions, that of $\mathrm{O}_{2}{ }^{+}$and that of ${ }^{64} \mathrm{Zn}^{2+}$. The relative amount of the two contributions can easily be found by counting the heavier $\mathrm{Zn}^{2+}$ isotopes contained in the peaks between 33 and $34 \mathrm{amu}$. (ii) The peak at $16 \mathrm{amu}$ can be attributed either to $\mathrm{O}_{2}{ }^{2+}$ or to $\mathrm{O}^{1+}$. However, the ratio of this peak to the peak at $32 \mathrm{amu}$ does not significantly change as a function of the charge state ratio $\mathrm{Zn}^{2+}$ / $\mathrm{Zn}^{1+}$. This is in agreement with the high second ionization energy of the $\mathrm{O}_{2}$ molecule (around $40 \mathrm{eV}^{49,50}$ compared with $17 \mathrm{eV}$ for $\mathrm{Zn}^{1+} \rightarrow \mathrm{Zn}^{2+51}$ ). Furthermore, atom probe analysis of ${ }^{18} \mathrm{O}$-enriched $\alpha-\mathrm{Fe}_{2} \mathrm{O}_{3}$ excluded the evaporation of the $\mathrm{O}_{2}{ }^{2+}$ 

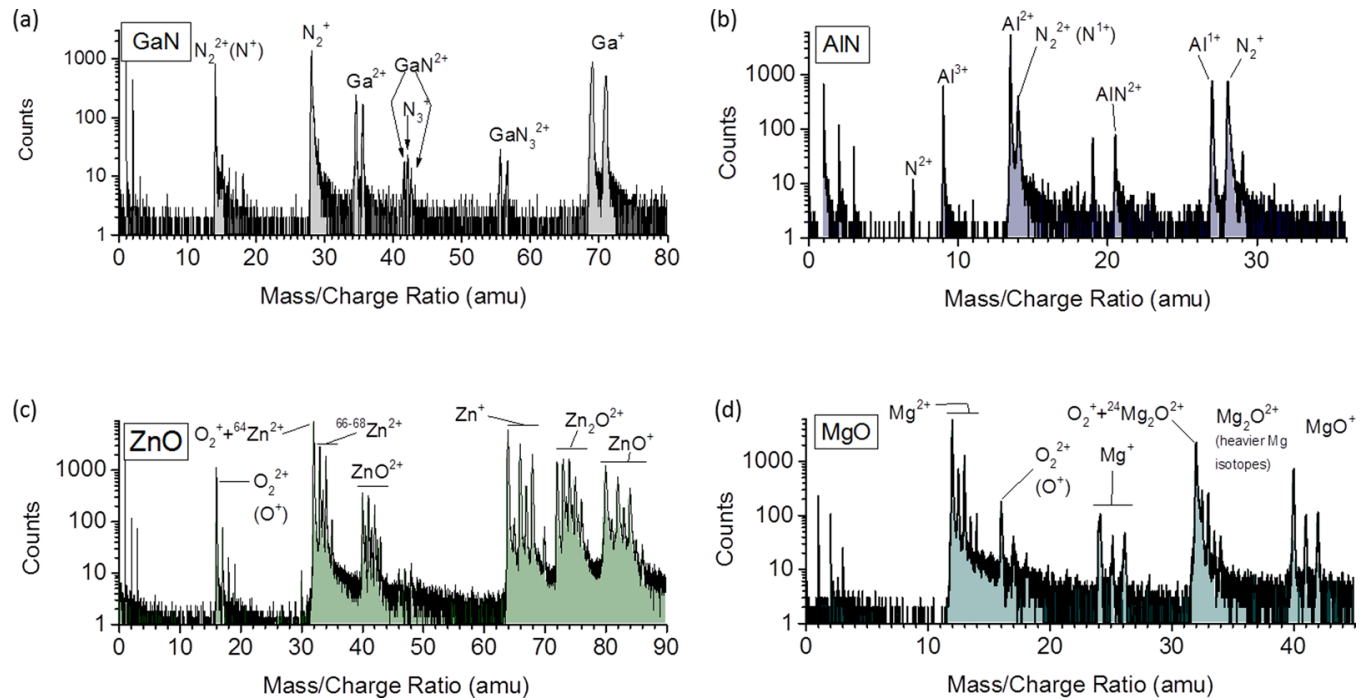

Figure 2. Mass spectra collected from different binary compound semiconductors. (a) GaN at $T=40 \mathrm{~K}$, laser pulse energy $E_{\text {las }}=0.7 \mathrm{~nJ}$, DC applied bias $V_{\mathrm{DC}}=6.0 \mathrm{kV}$, flux $\Phi=2.2 \times 10^{-3} \mathrm{At} / \mathrm{pls}$; (b) AlN at $T=20 \mathrm{~K}, E_{\text {las }}=6.3 \mathrm{~nJ}, V_{\mathrm{DC}}=10.2 \mathrm{kV}, \Phi=2.2 \times 10^{-3} \mathrm{At} / \mathrm{pls}$. (c) Nominally undoped $\mathrm{ZnO}$ at $T=50 \mathrm{~K}, E_{\mathrm{las}}=5 \mathrm{~nJ}, V_{\mathrm{DC}}=13.1 \mathrm{kV}, \Phi=2 \times 10^{-3} \mathrm{At} / \mathrm{pls} ;(\mathrm{d}) \mathrm{MgO}$ at $T=40 \mathrm{~K}, E_{\mathrm{las}}=5.0 \mathrm{~nJ}, V_{\mathrm{DC}}=8.6 \mathrm{kV}, \Phi=3 \times 10^{-3} \mathrm{At} / \mathrm{pls}$.

(a)

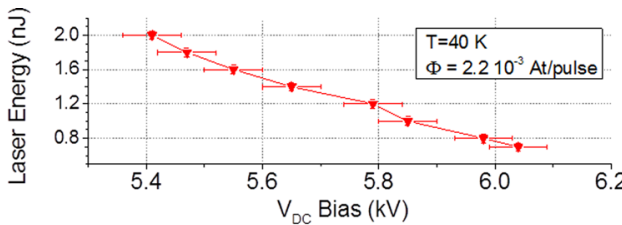

(b)

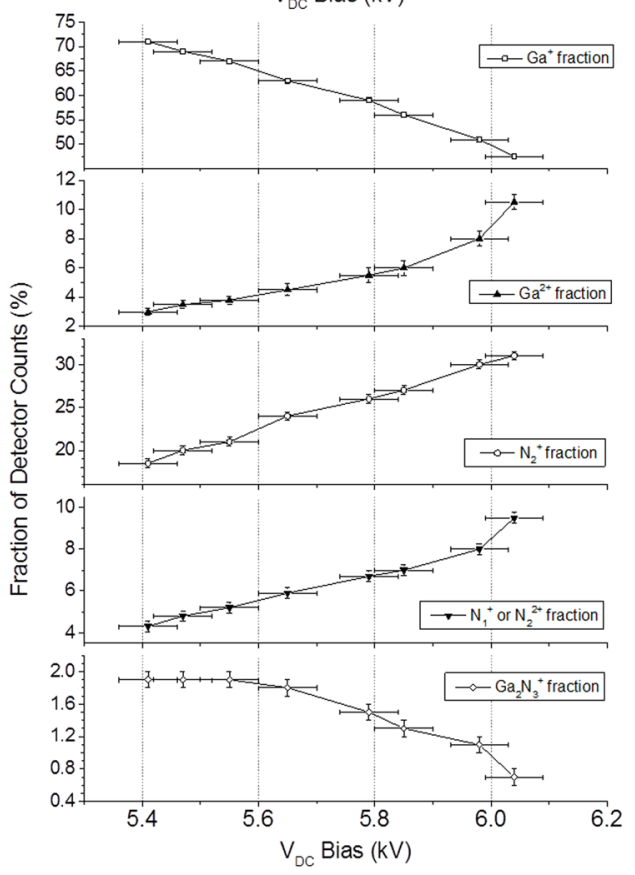

(c)

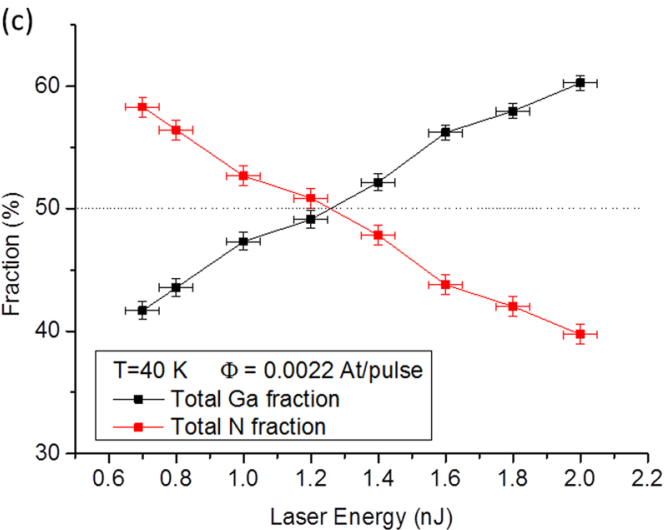

(d)

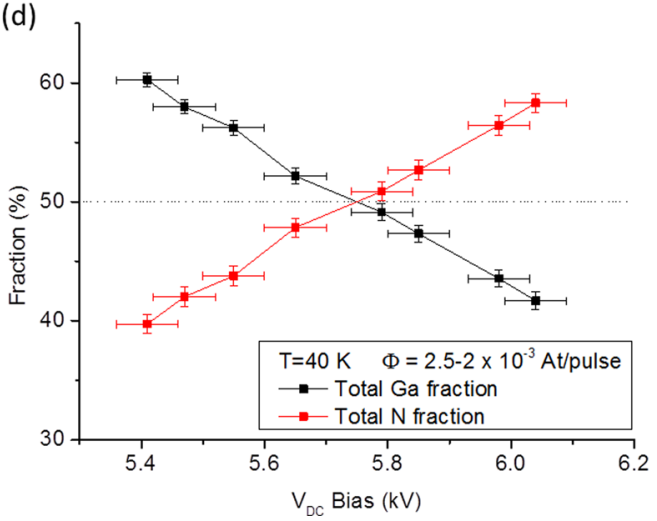

Figure 3. (a) Relationship between laser power and $V_{\mathrm{DC}}$ applied bias at the constant flux $\Phi=0.0022 \mathrm{At} / \mathrm{pls}$. (b) Dependence of the fraction (\%) of the different identified ion species on the applied bias $V_{\mathrm{DC}}$. From top to bottom: $\mathrm{Ga}^{1+}, \mathrm{Ga}^{2+}, \mathrm{N}_{2}^{+}, \mathrm{N}_{2}{ }^{2+}\left(\mathrm{N}^{+}\right)$, and $\mathrm{Ga}_{2} \mathrm{~N}_{3}$. $(\mathrm{Notice}$ : these are the fractions of detector counts assigned to a specific ion species: for the calculation of the total Ga and $\mathrm{N}$ fraction the multiplicity of a given atom in the ion is subsequently taken into account). (c) Total Ga and $\mathrm{N}$ fractions (\%) plotted as a function of the laser energy and (d) of the $V_{\mathrm{DC}}$ applied bias.

species, validating, on the contrary, the presence of $\mathrm{O}^{1+} .52$ Last, theoretical calculations performed by density functional theory (DFT) also predict the evaporation of $\mathrm{O}^{+}$species. ${ }^{53}$ The effect of the attribution of the peak at $16 \mathrm{amu}$ to $\mathrm{O}_{2}{ }^{2+}$ on the global composition measurement would be of increasing the relative amount of oxygen by about $5 \%$ over the whole explored range.
2.4.4. $\mathrm{MgO}$. An example of the $\mathrm{MgO}$ mass spectrum is shown in Figure 2(d). The main considerations for the labeling of the peaks are the following: (i) The peak at $32 \mathrm{amu}$ is related to two different contributions, i.e., that of $\mathrm{O}_{2}{ }^{+}$and that of $\left({ }^{24}\right.$ $\mathrm{Mg})_{2} \mathrm{O}^{1+}$. As for $\mathrm{ZnO}$, the separation of both contributions is made possible by counting the relative amount of peaks at 32.5 , 
(a)

\section{Study at constant DC bias}
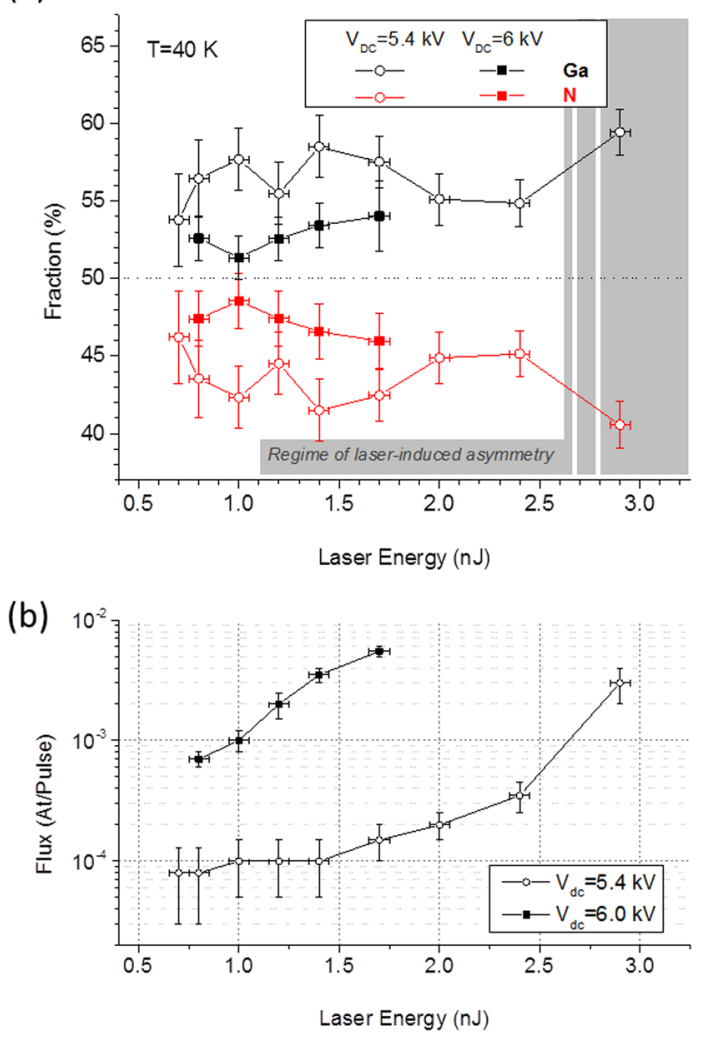

(c)

Study at constant laser energy

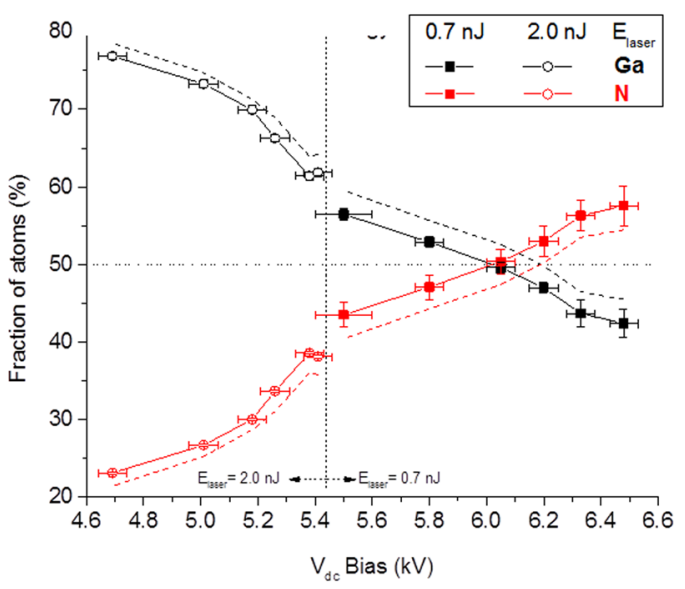

(d)

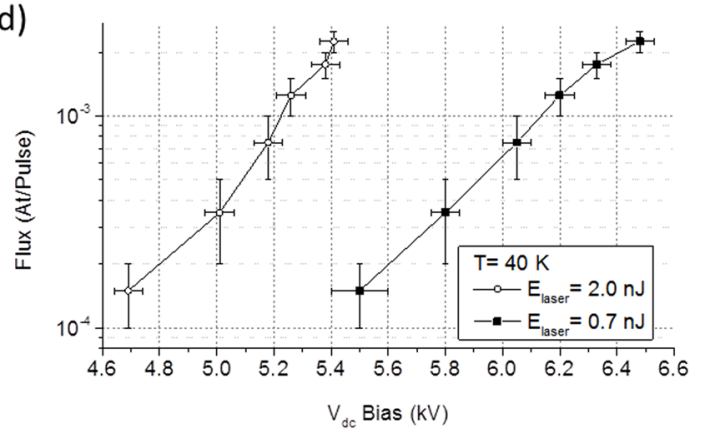

Figure 4. (a) Study at constant DC bias - Variation of the Ga (black symbols) and N (red symbols) fractions with the impinging laser energy for the two DC biases $V_{\mathrm{dc1}}=5.4 \mathrm{kV}$ (open circles) and $V_{\mathrm{dc} 2}=6.0 \mathrm{kV}$ (filled squares). The region in which a laser-induced asymmetry in the tip emission pattern is observed is highlighted in gray in the graph, above $2.5 \mathrm{~nJ}$. (b) Variation of the flux with the impinging laser energy for the two DC biases $V_{\mathrm{dc} 1}=5.4 \mathrm{kV}$ (open circles) and $V_{\mathrm{dc} 2}=6.0 \mathrm{kV}$ (filled squares). (c) Study at constant laser energy - Dependence of the total Ga (black symbols) and $\mathrm{N}$ (red) fractions (\%) on the applied bias. Empty and filled symbols correspond to data acquired using a laser energy of $E_{1}=0.7 \mathrm{~nJ}$ and $E_{2}=2.0 \mathrm{~nJ}$, respectively. Note that the dashed lines represent the composition obtained under the hypothesis that the peak at 14 amu is $\mathrm{N}^{+}$instead of $\mathrm{N}_{2}{ }^{2+}$. (d) Study at constant laser energy - Variation of the flux with the applied bias for the two laser energies $E_{1}=0.7 \mathrm{~nJ}$ (filled squares) and $E_{2}=2.0 \mathrm{~nJ}$ (empty circles).

33, and $34 \mathrm{amu}$, related to $\mathrm{Mg}_{2} \mathrm{O}^{1+}$ molecules containing at least one $\mathrm{Mg}$ atom with mass larger than $24 \mathrm{amu}$. (ii) Concerning the peak at $16 \mathrm{amu}$, the same considerations as in $\mathrm{ZnO}$ apply, and the attribution as $\mathrm{O}^{1+}$ was considered as the more likely. Attributing the peak at $16 \mathrm{amu}$ to $\mathrm{O}_{2}{ }^{2+}$ would raise the total fraction of oxygen by about $2 \%$, consistent with previous results. $^{25}$

2.5. "Standard" Composition Analysis of GaN at Constant Flux. During an APT analysis, parameters such as temperature, laser pulse energy, and applied DC voltage can be tuned in order to work in the most convenient conditions. It is also customary to work at constant flux throughout the analysis. Previous studies on $\mathrm{GaN}^{22,23}$ and $\mathrm{MgO}^{25}$ nonstoichiometric evaporation have generally expressed the compositional biases in terms of laser energy, with the notable exception of the work by Agrawal et al., who also expressed the results as a function of the $\mathrm{Ga}^{1+} / \mathrm{Ga}^{2+}$ global charge state ratio. ${ }^{20}$ In this section, we show in detail how the composition measurement in GaN can be studied at constant flux. However, this "standard" approach has a major limitation: the simultaneous variation of laser pulse energy and DC applied bias makes it impossible to decide whether the measurement is influenced by one or both of these parameters. This is illustrated in Figure 3(a) where the laser power and the DC applied bias vary in order to obtain an evaporation flux $\Phi=0.0022 \mathrm{At} / \mathrm{pls}$. The fraction of events with definite attribution in the mass spectrum $\left(\mathrm{Ga}^{1+}, \mathrm{Ga}^{2+}, \mathrm{N}_{2}^{+}, \mathrm{N}_{2}{ }^{2+}\right.$ $\left(\mathrm{N}^{+}\right.$), and $\mathrm{Ga}_{2} \mathrm{~N}_{3}$ from top to bottom) is reported in Figure 3(b) as a function of the applied bias. Once the multiplicity of each element per event is taken into account, it becomes possible to calculate the total $\mathrm{N}$ and $\mathrm{Ga}$ fractions as a function either of the laser pulse energy, as reported in Figure 3(c), or of the DC applied bias, as in Figure 3(d). These results show that the composition of $\mathrm{GaN}$ measured at high bias and low laser energy is $\mathrm{N}$-rich and Ga-rich at low bias and high laser energy.

2.6. Composition Analysis of $\mathrm{GaN}$ at Constant Laser Pulse Energy and at Constant DC Bias. In order to discriminate whether the biases in the measured composition are due to the laser pulse energy, to the DC bias, or to a combination of both, we performed further series of measurements on GaN, i.e., two series of measurements at constant laser pulse energy and two series of measurements at constant DC applied bias, with the flux varying consequently.

The plots reported in Figure 4(a) refer to the composition measured under constant bias conditions. The interesting point to notice here is that in this regime the laser does not significantly influence the compositional bias, all experimental points yielding consistent values within the error bars. When the laser begins to induce a departure from the composition of $55 \% \mathrm{Ga}$ and $45 \% \mathrm{~N}$ in the series acquired at low DC bias, this is also accompanied by the appearance of an asymmetric pattern 
in the tip emission. The regime in which laser heating produces an asymmetric pattern is extremely interesting, ${ }^{22}$ but was not analyzed in detail in this study, for two main reasons: (i) in this regime severe tip deformation may take place, which would bias the data acquired in successive experiments before a sufficiently symmetric shape is recovered, and (ii) in this regime, the induced asymmetry is not suitable for obtaining reliable atom probe data, especially applied to the problem of dopant distribution and heterostructure reconstruction. Figure 4(b) illustrates how the flux depends on the applied laser energy for the two different values of $V_{\mathrm{DC}}$.

The effect of the DC bias on the stoichiometry was studied in two regimes: a low laser energy regime, with the laser set at 0.7 $\mathrm{nJ}$, and a high laser energy regime, with the laser set at $2.0 \mathrm{~nJ}$. The total fractions of $\mathrm{Ga}$ and $\mathrm{N}$ as a function of the applied bias are reported in Figure 4(c), while the variation of the flux as a function of the bias is reported in Figure 4(d). One can notice (i) that the behavior is similar to what was obtained during the measurements at constant flux plotted as a function of applied bias: Ga is overestimated at low DC bias, whereas $\mathrm{N}$ is slightly overestimated at high DC bias and (ii) the laser has very little influence on the behavior: the two curves at different laser energy follow the same trend, and there is no clear discontinuity at the point where the laser energy passes from one value to the other. The reader should consider that in this point, which corresponds in the plot below to the passage between 5.4 and $5.5 \mathrm{kV}$, the flux varies by a factor of $\sim 15$.

Figure 4(c) also shows how the obtained fractions change according to the assumption on the ion detected at $14 \mathrm{amu}$. All experimental points refer to the assumption that this peak is $\mathrm{N}_{2}{ }^{2+}$. As is visible from the figure, passing from this assumption to the one that the peak at $14 \mathrm{amu}$ (dashed lines) is $\mathrm{N}^{+}$does not change the main trend of the composition and only slightly shifts the DC bias for which the crossover of the fractions occurs.

2.7. Applied Protocol of Analysis. The data displayed in the two previous sections point out the following:

(a) Composition measurements performed at constant flux may be useful to find a set of parameters for which the global composition is stoichiometric. However, due to the simultaneous variation of laser pulse energy and DC bias, they do not indicate whether the biases are due to the absorbed laser energy or to the surface electric field.

(b) In the case of $\mathrm{GaN}$, additional series of measurements performed at constant laser pulse energy and at constant DC bias indicate that the measured composition is rather independent of the absorbed laser energy, while it exhibits a strong dependence on the applied DC bias. The main physical factor inducing compositional biases in this material is therefore the surface electric field.

(c) If the measurement of the composition of a given semiconducting compound yields parameter-dependent results, more series of measurements performed at constant flux, constant laser energy, and constant DC bias should be performed in order to understand which physical parameter plays the major role.

In this work, therefore, we adopted the following protocol of measurement, which we applied to field emission tips of different materials.

(i) A series of measurements at constant flux were performed. As the laser pulse energy was changed for each acquisition, the DC voltage on the tip was allowed to change in order to restore the flux. After reaching a stationary regime, 100 to $200 \mathrm{kAtoms}$ (excluding noise and hydrogen-related species) were collected-this quantity was found to be sufficient for a robust statistical analysis while not significantly modifying the shape of conical tips.

(ii) One or two series of measurements at constant DC bias: in this case the laser pulse energy is varied, and the flux is allowed to vary consequently. The higher limit for the laser pulse energy was either the onset of tip shape modification due to surface diffusion or that corresponding to a flux of 0.05 ions/ pulse, while the lower limit was set by a flux equal to $10^{-4} \mathrm{At} /$ pls. The total number of identified atoms per data set was 25$200 \times 10^{3}$.

(iii) One or more series of measurements at constant laser pulse energy: in this case the DC bias is varied, and the flux is allowed to vary consequently. The DC bias higher limit was either $14 \mathrm{kV}$ or the value corresponding to a flux of $0.05 \mathrm{At} / \mathrm{pls}$, while the lower limit corresponded to a flux of $10^{-4} \mathrm{At} / \mathrm{pls}$. FIB-milled tips are typically developed in the atom probe before the application of the protocol in order to avoid compositional biases due to the presence of implanted $\mathrm{Ga}$ ions. The total number of identified atoms per data set was $25-200$ $\times 10^{3}$

2.8. Representation of Composition As a Function of the Charge State Ratio of the Metallic Element. Once the protocol of measurements has been applied, an important question concerns the way of representing the results in order to correctly interpret them and possibly to compare them to the results obtained in other materials or by other operators. As in the case of $\mathrm{GaN}$, the result for all other materials analyzed in this study points out that the measured composition does not depend on the laser energy. It would be therefore misleading to represent the data as a function of this parameter. Another choice could be the pulse fraction. This quantity corresponds to the quantity $\mathrm{PF}=1-V_{\mathrm{DC}} / V_{0}$, where $V_{0}$ is the evaporation potential found by extrapolating to zero laser power the nearly linear $V_{\mathrm{DC}} / E_{\text {las }}$ relationship that can be found in a series of measurements performed at constant flux, an example of which is reported in Figure 3(a). However, we notice that the pulse fraction is defined for a given flux, and the ensemble of the data (specifically those collected at constant laser and at constant DC bias) is collected at different fluxes. The pulse fraction is thus not a good parameter for a coherent representation of the whole data set.

Alternatively, the applied DC bias could be used. This appears as a judicious choice for bias-dependent data, but it must be mentioned that due to tip blunting during the analysis conical tips may exhibit strong variations either of the applied bias at constant flux or of the surface field at constant bias. The same consideration applies to different tips with different sizes.

In our opinion, the most convenient choice for the representation of composition measurements is as a function of the $\mathrm{Me}^{2+} / \mathrm{Me}^{1+}$ charge ratio, where by $\mathrm{Me}$ we indicate the metallic element of the compound. This has several advantages with respect to other possible representations:

(i) The quantity $\mathrm{Me}^{2+} / \mathrm{Me}^{1+}$ is related to the surface field. This relationship might be made quantitative through the postionization statistics introduced by Kingham. ${ }^{48}$ In principle, the phenomenon of postionization takes place at a distance of several angstroms from the sample surface and should be nearly independent of the material in which the atom was found if the evaporation takes place in the singly charged state. The charge state abundances of the evaporated ions are thus a function of the surface field. Experimentally, the $\mathrm{Me}^{2+} / \mathrm{Me}^{1+}$ charge state 
(a)

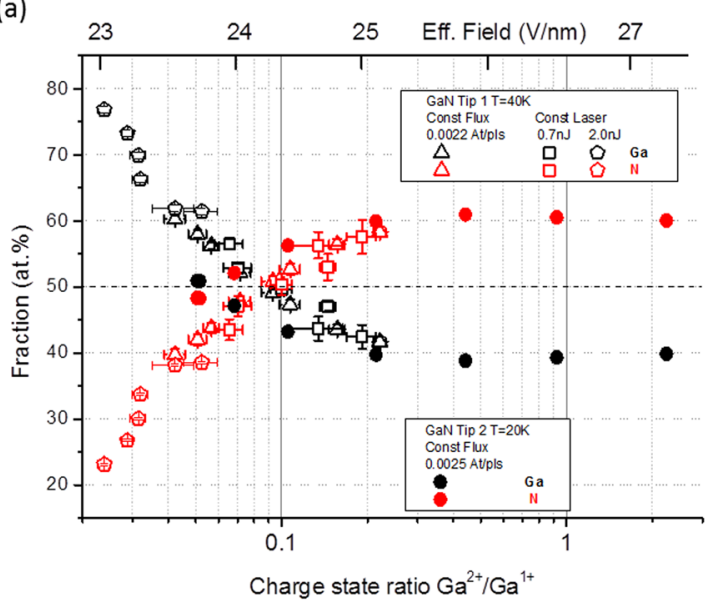

(c)

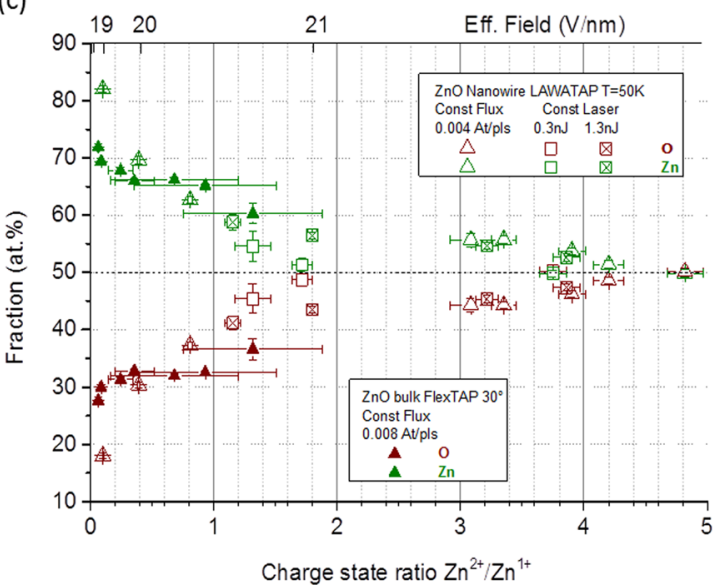

(b)

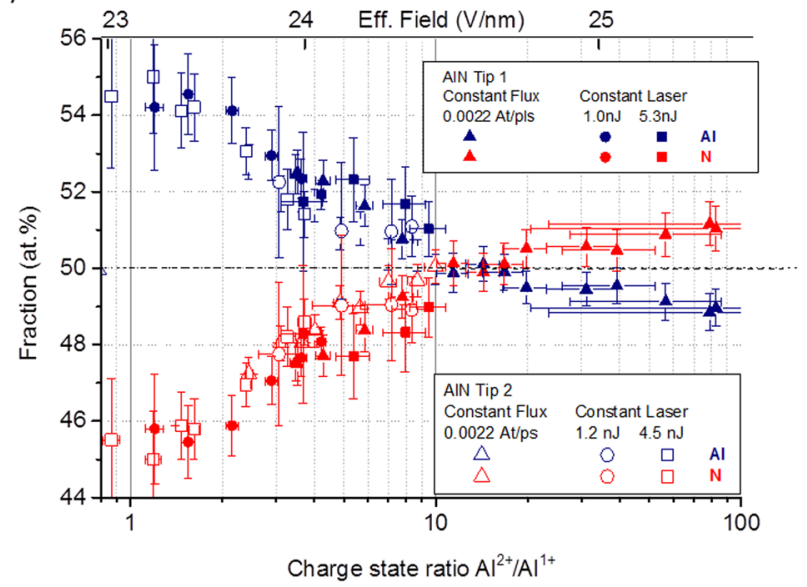

(d)

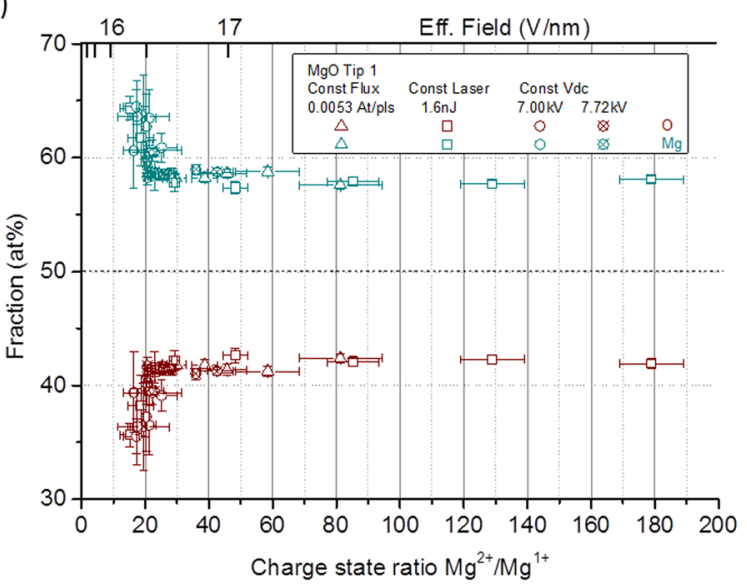

Figure 5. Average atomic fractions measured by the UV laser-assisted atom probe in binary compound semiconductors as a function of the average charge state ratio $\mathrm{Me}^{2+} / \mathrm{Me}^{1+}$, where Me indicates the metallic element of the binary compound. (a) Data for two GaN tips oriented along the $c$-axis, the first one measured at $T=40 \mathrm{~K}$ applying the full protocol (empty symbols) and the second one at $T=20 \mathrm{~K}$ at constant flux only (full circles). (b) Data for two AlN tips of different apex radii $\left(r_{1}=50 \mathrm{~nm}, r_{2}<30 \mathrm{~nm}\right)$ at $T=20 \mathrm{~K}$. Notice that the data are plotted versus an $x$ log scale for clarity. (c) Data for two undoped $\mathrm{ZnO}$ tips: the first is a nanowire analyzed in the LAWATAP at $T=50 \mathrm{~K}$, while the second one is extracted from bulk material and analyzed in the FlexTAP at $T=80 \mathrm{~K}$ at constant flux only. (d) Data for FIB-milled MgO tips measured at $T=40 \mathrm{~K}$ (for the sake of completeness, the analysis of a second tip is reported in Figure S-6 in the Supporting Information). For all graphs, the effective surface field is reported in the top abscissa scale.

ratio is an increasing function of the applied DC bias, provided the tip shape remains nearly unchanged during the analysis, as illustrated in Figure S-2 in section S-1.e of the Supporting Information. By inverting the charge abundance/field relationship, it becomes then possible to calculate an effective surface field. However, this effective field should only be considered as an estimate of the actual surface field. First, it is known that the Kingham charge state statistics is based on approximations that make its application to 3D tips questionable. ${ }^{54,55}$ Furthermore, our data occasionally show features which are not compatible with the Kingham statistics, such as, for instance, the simultaneous presence of the charge states $\mathrm{Al}^{1+}, \mathrm{Al}^{2+}$, and $\mathrm{Al}^{3+}$ in AlN.

(ii) For analyses performed on the same tip sample, the $\mathrm{Me}^{2+} / \mathrm{Me}^{1+}$ charge state ratio allows for a robust evaluation of the composition trends even in the case where the tip shape significantly changes during the analysis.

(iii) For analyses performed on different tips of the same material, the $\mathrm{Me}^{2+} / \mathrm{Me}^{1+}$ charge state ratio is the only parameter that allows for a direct comparison, which would be extremely difficult to achieve considering parameters such as DC voltage, laser pulse energy, or laser energy-related pulse fraction. These experimental parameters are indeed related to the electric surface field and to the absorbed energy per pulse through the geometrical shape of the tip, which is difficult to monitor during an APT experiment.

(iv) The $\mathrm{Me}^{2+} / \mathrm{Me}^{1+}$ charge state ratio can be defined either as a global or as a local quantity, as it can be calculated either as an average or point by point by defining sampling pixels on the 2D detector. It thus becomes possible to establish microscopic relationships between the local surface field and the locally measured composition.

(v) Finally, the $\mathrm{Me}^{2+} / \mathrm{Me}^{1+}$ charge state ratio allows comparing analyses performed on different instruments and by different users.

\section{RESULTS}

3.1. Measurement of the Composition of Binary Compound Semiconductors. In Figure 5 we report the average measured fraction of the Me elements III (II) and $\mathrm{N}$ (O) in the analyzed materials after applying the protocol described in section 2.7. Through the measurements performed 
(a)

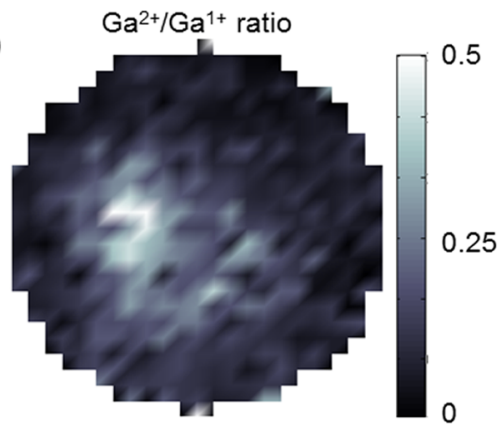

(b)

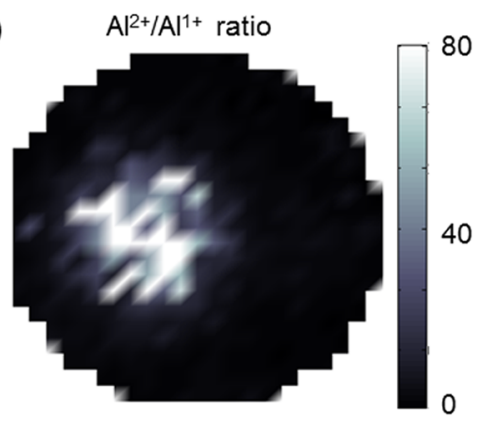

(c)

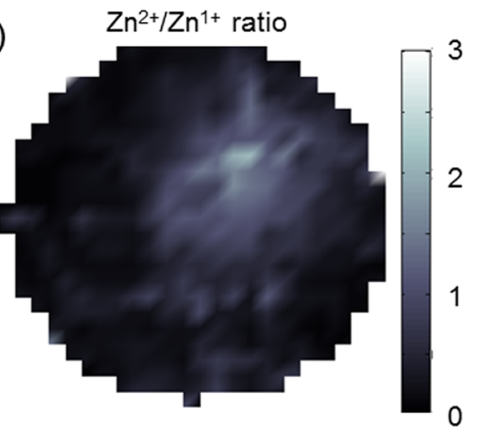

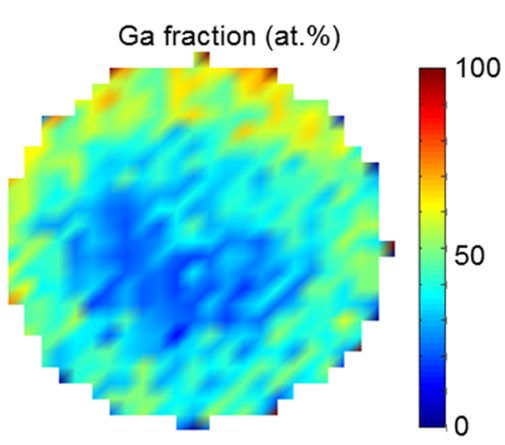

100
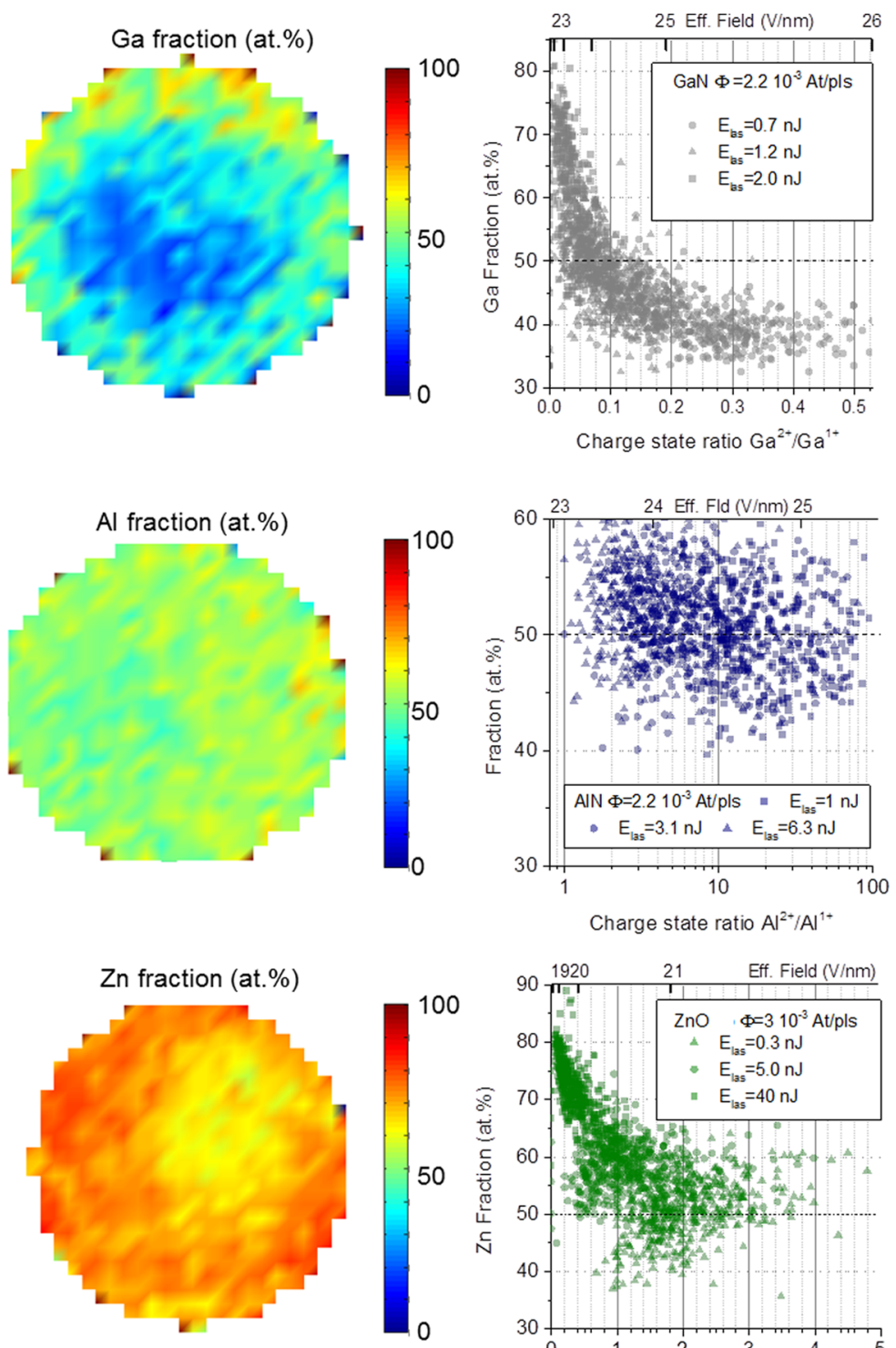
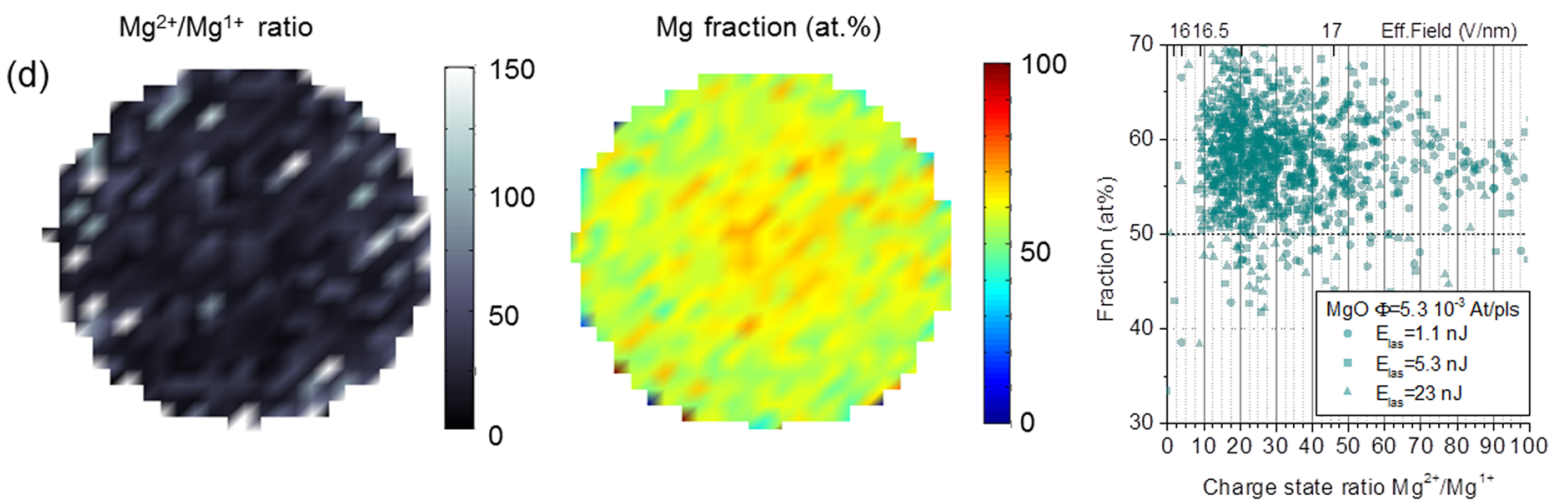

Figure 6. Correlation between surface electric field and measured composition visualized at a microscopic scale through detector hitmap statistics. The figures on the left-hand side show the spatial distributions of the charge states $\mathrm{Me}^{2+} / \mathrm{Me}^{1+}$, assumed as an indicator of the surface electric field. The figures in the central column display the spatial distributions of the fraction of the metallic element. The graphs on the right-hand side report the correlation between the $\mathrm{Me}^{2+} / \mathrm{Me}^{1+}$ charge state and the fraction of metallic element measured locally on the detector for three different sets of experimental parameters at constant flux. (a) $\mathrm{GaN}$ at $T=40 \mathrm{~K}$, flux $\Phi=2.2 \times 10^{-3} \mathrm{At} / \mathrm{pls}$ and (detector hitmaps) $E_{\text {las }}=1.2 \mathrm{~nJ}$, (correlation plot) $E_{\text {las }}$ $=0.7,1.2$, and $2.0 \mathrm{~nJ}$. (b) AlN at $T=20 \mathrm{~K}$, flux $\Phi=2.2 \times 10^{-3} \mathrm{At} / \mathrm{pls}$ and (detector hitmaps) $E_{\mathrm{las}}=6.3 \mathrm{~nJ}$, (correlation plot) $E_{\text {las }}=1.0,3.1$, and 6.3 $\mathrm{nJ}$. Notice that the data are plotted versus an abscissa log scale for clarity: (c) $\mathrm{ZnO}$ at $T=50 \mathrm{~K}$, flux $\Phi=3 \times 10^{-3} \mathrm{At} / \mathrm{pls}$ and (detector hitmaps) $E_{\text {las }}$ $=0.3 \mathrm{~nJ}$ (correlation plot), $E_{\mathrm{las}}=0.3,5.0$, and $40 \mathrm{~nJ}$. (d) $\mathrm{MgO}$ at $T=40 \mathrm{~K}$, flux $\Phi=5 \times 10^{-3} \mathrm{At} / \mathrm{pls}$, (detector hitmaps) $E_{\text {las }}=5.3 \mathrm{~nJ}$, (correlation plot) $E_{\mathrm{las}}=1.1,5.3$, and $23 \mathrm{~nJ}$. 
at constant DC bias we could ascertain that in the explored intervals of parameters the measured composition does not depend, or depends very weakly, on the laser pulse energy. This conclusion confirms that whatever the materials and tips analyzed all points relative to the measured composition as a function of the $\mathrm{Me}^{2+} / \mathrm{Me}^{1+}$ charge state ratio nearly fall on the same curve independently of the series of measurements being acquired at constant flux, DC bias, or laser power. It is worth noticing that this behavior is not general for all semiconductors and dielectrics. The specific role played by the laser energy has for instance been assessed in narrower-bandgap semiconductors, such as $\mathrm{GaSb}^{21}$ Figure 6 illustrates the spatial distribution of the $\mathrm{Me}^{2+} / \mathrm{Me}^{1+}$ charge state ratio (left column) and the microscopic measurement of the Me element fraction (mid column) within a selected data set containing $\sim 10^{5}$ atoms collected at fixed experimental parameters. The correlation plots of the two distributions are reported in the right-hand side column for three different data sets, in order to show that the microscopic behavior reproduces the average data shown in Figure 5. The effective fields shown on the upper abscissa axes are derived by the charge state ratios according to the Kingham model.

For all analyzed materials, there is a general common feature: when the field is low, there is an excess of metallic element, which tends to be reduced at high field. This behavior, recently confirmed in $\mathrm{GaN}$ and $\mathrm{MgO}$, has been ascribed to the diffusion of neutral $\mathrm{N}_{2}$ and $\mathrm{O}_{2}$ molecules, ${ }^{20,22,23,25}$ the formation of which would be possible on the tip surface exposed to the field. These molecules would not be detected. The diffusion of neutral $\mathrm{O}$ down the tip shaft and the subsequent formation of weakly bound $\mathrm{O}_{2}$ molecules likely to desorb have recently been suggested by density functional theory calculations performed on small $\mathrm{MgO}$ clusters immersed in an external field and neglecting the presence of laser illumination. ${ }^{53}$ Such molecules would be progressively postionized and become detectable when the surface field increases. This mechanism cannot be proven by our data, but it would lead to an increase in the measured fraction of $\mathrm{N}$ or $\mathrm{O}$ with the surface field, consistent with our measurements. However, another mechanism adds to the postionization of neutral molecules, i.e., the preferential evaporation of the metallic element atoms. This phenomenon leads to a decrease in the measured fraction of $\mathrm{Me}$ atoms with increasing field and, simultaneously, to an increase in the fraction of background noise in the mass spectra. The role of the field and the absence of laser effects are confirmed by the detector hitmaps shown in Figure 6. For each material, the high field regions (corresponding to a high $\mathrm{Me}^{2+} / \mathrm{Me}^{1+}$ charge state ratio) correspond to a lower measured fraction of the $\mathrm{Me}$ element.

These features are common to the sets of data shown in Figures 5 and 6 , but the behaviors of the different materials differ significantly.

3.1.1. GaN. GaN, whose behavior is illustrated in Figure $5(\mathrm{a})$, exhibits a large excursion of the measured $\mathrm{Ga} / \mathrm{N}$ fractions with the surface field. The measured $\mathrm{Ga}$ fraction passes from $80 \%$ at low field to approximately $40 \%$ at high field. At $T=40$ $\mathrm{K}$, an average stoichiometric behavior can be achieved when the $\mathrm{Ga}^{2+} / \mathrm{Ga}^{1+}$ ratio is around 0.1 , corresponding to an effective surface field of $\sim 24.3 \mathrm{~V} / \mathrm{nm}$. The microscopic analysis performed on a tip oriented on the $c$-axis and reported in Figure 6(a) shows that the high-field regions concentrate around the $c$-pole. The correlation plot, furthermore, indicates that even if the experimental conditions are chosen in order to obtain an average stoichiometric composition the microscopic composition is not stoichiometric, as the tip shape is never perfectly spherical and the surface field is never homogeneous. The correlation plot of Figure 6(a) and the measurements performed on a second tip at $T=20 \mathrm{~K}$ at constant flux (full circles in Figure 5(a)) also indicate that the high-field composition tends to saturate to $40 \%$ of $\mathrm{Ga}$ and $60 \%$ of $\mathrm{N}$. This means that at high field preferential evaporation affects $\mathrm{Ga}$ and $\mathrm{N}$ at a fixed ratio. As will be discussed in a following section, the amount of lost atoms can be estimated either by quantifying the background noise or with the help of InGaN quantum wells used as a marker for a correct volume reconstruction. These latter data indicate that for nearly stoichiometric conditions about one over two detectable atoms is lost.

3.1.2. AIN. The behavior of AlN is reported in Figures 5(b) and 6(b). Two tips with different apex radii were analyzed, yielding consistent results. Like GaN, AlN appears N-poor at low field (or in low-field regions) and $\mathrm{N}$-rich at high field (or in high-field regions). A stoichiometric composition can be achieved when $\mathrm{Al}^{2+} / \mathrm{Al}^{1+} \sim 10$, corresponding to an effective surface field of around $24.4 \mathrm{~V} / \mathrm{nm}$, quite similar to that for which a stoichiometric composition is achieved in GaN. It is interesting to notice that this value is in contrast to the observation of $\mathrm{N}^{2+}$ species in AlN, which suggests that AlN was analyzed at higher surface fields. However, the measured composition of AlN is more robust than that of $\mathrm{GaN}$, remaining much closer to the stoichiometric value in the whole interval of explored surface field. From the available data, it is not possible to conclude if the high-field composition saturates to a fixed value or not.

3.1.3. ZnO. The behavior of nominally undoped $\mathrm{ZnO}$ reproduces some features of the behavior of $\mathrm{GaN}$ : the measured composition is Zn-rich (over 80\%) at low field and tends to a stoichiometric value at high field (corresponding to an effective field higher than $21.2 \mathrm{~V} / \mathrm{nm}$ ), as reported in Figures 5(c) and 6(c). The data on the plot of Figure 5(c) are related to two tips analyzed under different conditions. The first one is a nanowire analyzed along the $c$-axis in the LAWATAP at $T=$ $50 \mathrm{~K}$, while the second one was extracted from a polycrystalline bulk sample (the exact crystal orientation is unknown) and analyzed in a FlexTAP at $T=80 \mathrm{~K}$. This second tip was only analyzed at constant flux $\Phi=0.008 \mathrm{At} / \mathrm{pls}$. It is interesting to notice that the two tips yield consistent results despite all the different experimental parameters adopted. In the case of the FlexTAP, the uncertainty on the charge state ratio is larger due to the larger portion of surface imaged in this instrument. The detector hitmap analysis of Figure 6(c) issued by the nanowire sample also indicates that a lower $\mathrm{Zn}$ amount is measured in high-field regions of the tip surface. However, we cannot conclude whether the $\mathrm{Zn}$ and $\mathrm{O}$ fractions cross at high field or if they asymptotically tend to $50 \%$. Finally, it is worth noticing that in $\mathrm{ZnO}$ some residual dependence of the measured composition on the laser energy can be assessed, as shown in the Supporting Information (Figure S-4).

3.1.4. $\mathrm{MgO}$. $\mathrm{MgO}$ also exhibits specific features. As reported in the plot in Figure 5(d), the measured $\mathrm{Mg}$ fraction at low field is around $65 \%$. In the case of $\mathrm{MgO}$ and in the explored interval of parameters, the $\mathrm{Mg}$ and $\mathrm{O}$ fractions tend to $58 \%$ and $42 \%$, respectively. This behavior is confirmed for another tip, the data relative to which are shown in the Supporting Information, Figure S-6. It is interesting to notice that the microscopic behavior of the tip shown in Figure 6(d) indicates a weak 

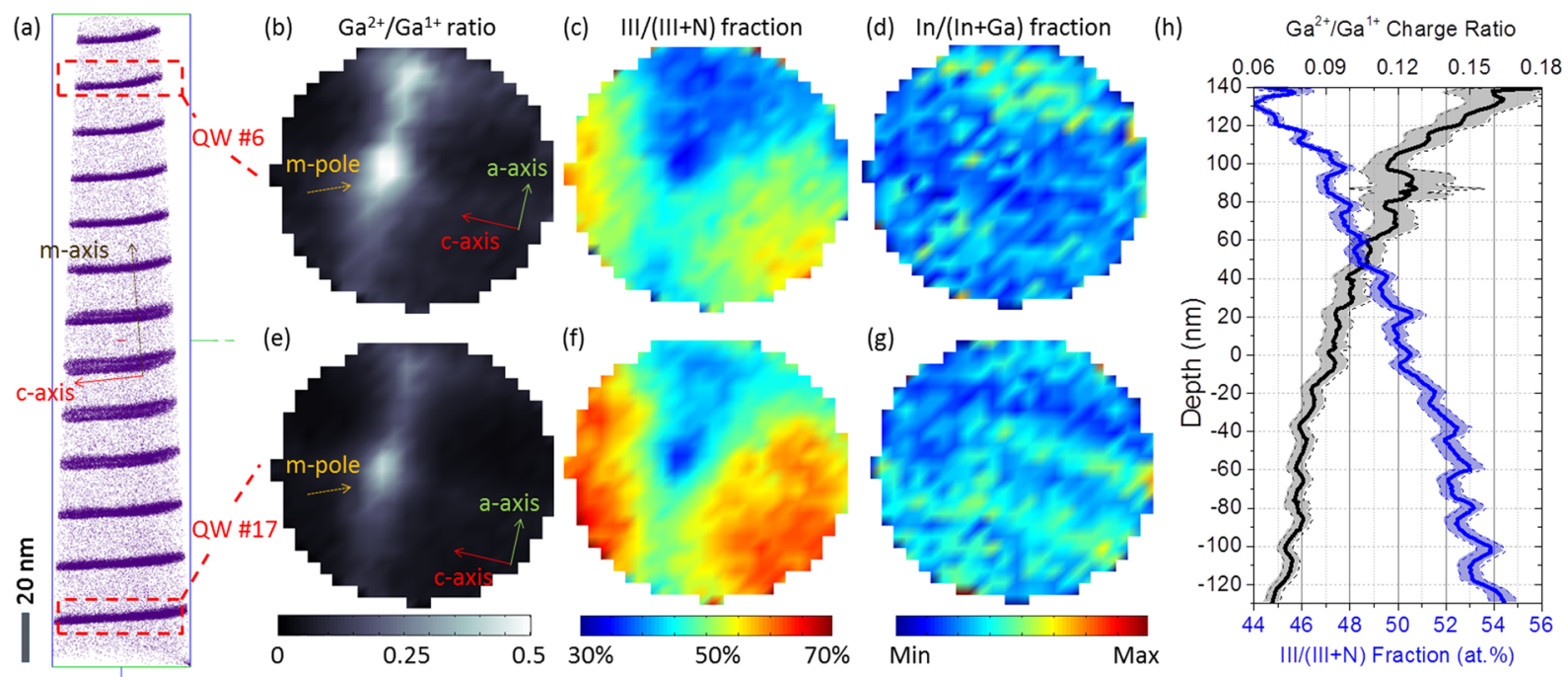

Figure 7. Study of the spatial correlation between the measured Ga charge ratio, the measured fraction of element III, and the $x$ InN alloy fraction in an $\operatorname{In}_{x} \mathrm{Ga}_{1-x} \mathrm{~N} / \mathrm{GaN}$ multiple quantum well system analyzed at $T=20 \mathrm{~K}$. (a) $3 \mathrm{D}$ reconstruction of atom probe data-the position of In atoms only is shown for clarity. $(b, c, d)$ Detector hitmaps averaged from the red box in (a) containing QW\#6, showing the $\mathrm{Ga}^{2+} / \mathrm{Ga}^{1+}$ charge ratio distribution, the measured fraction of element III, and the reduced $x \mathrm{InN}$ alloy fraction, respectively. (e,f,g) The same detector hitmap analysis from the box containing QW\#17. (h) Plot illustrating the correlation between the average $\mathrm{Ga}^{2+} / \mathrm{Ga}^{1+}$ ratio (black line, top $x$-scale) and the average measured element III fraction (blue line, bottom $x$-scale), as a function of the depth of analysis; the dashed lines and the filled area indicate the variance of the hitmap data. The representation of the whole data set is available as a video in the Supporting Information.

correlation of high-field regions and lower $\mathrm{Mg}$ content, but here the high-field regions are distributed close to the edge of the detector. This could be due to the specific shape acquired by the tip-with a larger radius of curvature at the apex and smaller at the apex-shank edge-during the analysis, as has already been observed in previous works. ${ }^{25}$

3.2. Measurement of Alloy Fraction in III-N Heterostructures. The results exposed in the previous section point out that in the case of binary III-N materials it is difficult to obtain a stoichiometric measurement of the atoms during a whole atom probe run and at each point of the analyzed volume. Moreover, in the particular case of $\mathrm{GaN}$ the inhomogeneous distribution of the surface electric field on the tip induces strong biases in the measured concentrations. As already remarked in a previous work, the measurement of the correct stoichiometric ratio of element III to $\mathrm{N}$ should not be the ultimate target. ${ }^{23}$ On one hand, it is known that III-N compound semiconductors are intrinsically stoichiometric; i.e., there is always an element III atom for each $\mathrm{N}$ atom, with allowed deviations much smaller than $0.1 \%$ and maximal deviations of the order of $1 \%$ in $\mathrm{GaN}^{56}$ Second, the most interesting cases for which atom probe can be a useful tool for the characterization of these materials at the nanoscale is the quantification of dopants and the $3 \mathrm{D}$ reconstruction of the composition and geometry of heterostructures. In the following, we address the critical point of the correct quantification of alloy fractions in nanoscale heterostructures including ternary III-N alloys. Riley et al. already showed that despite the bias in the measurement of the element III fraction there is no bias induced in the measurement of alloy fractions in an InGaN quantum well (QW) with random alloy distribution. These authors worked at experimental conditions yielding a $\mathrm{Ga}^{2+} / \mathrm{Ga}^{1+}$ ratio between 0.2 and 0.5 , corresponding to N-rich conditions 24. Other experimental APT studies performed on InGaN multiple QW systems with low laser pulse energy, also support this conclusion. ${ }^{57-64}$ An answer to the question about the robustness of alloy fraction measurement can be based on the correlation of the alloy fraction measurement with the surface field indicators. In the following, we provide strong evidence for a robust alloy fraction measurement in different III-N alloys, namely InGaN and InAlN.

3.2.1. InGaN. The data relative to $\mathrm{InGaN}$ were collected from a field-emission tip containing a 20-fold InGaN/GaN multi-QW system extracted from a microwire grown by metalorganic chemical vapor deposition (MOCVD). A part of the data relative to this tip, along with the analysis of the structural properties of QWs by APT and their correlation with structural and crystallographic data from high-resolution scanning transmission electron microscopy (HR-STEM) characterization and the optical properties measured by microphotoluminescence $(\mu \mathrm{PL})$ spectroscopy, have been published in ref 45 . The tip was analyzed by APT at $20 \mathrm{~K}$, under an UV laser illumination with $0.3 \mathrm{~nJ}$ pulses, at a constant flux $\Phi=0.0055 \pm$ $0.0005 \mathrm{At} / \mathrm{pls}$. During the analysis, about 27 million atoms were collected, corresponding to the reconstructed volume shown in Figure 7(a) (for clarity's sake only In atoms are displayed). The electron microscopy data showed that the tip axis nearly coincides with one of the $m$-axes of the hexagonal wurtzite crystal and also allowed us to assess the orientation of the $c$ and $a$-axes in the atom probe reconstructed volume. Furthermore, the HR-STEM data have been used for obtaining the best possible volume reconstruction, based on the presence of QWs as markers for the depth coordinate. ${ }^{45}$ It is worth noticing that the support of the STEM data sets strong constraints on the APT geometrical reconstruction parameters (the cone angle and initial tip radius algorithm was used for this data $\operatorname{set}^{65}$ ), and the best reconstruction could be obtained by setting a detector efficiency as low as 0.3 , which is about onehalf of the upper theoretical limit for the microchannel detector plate. ${ }^{66}$ This indicates that about one-half of the sample atoms are not detected and are lost either as preferentially or as neutrally evaporated species. In the reported analysis, 12 over 20 QWs could be reconstructed. 
(a)
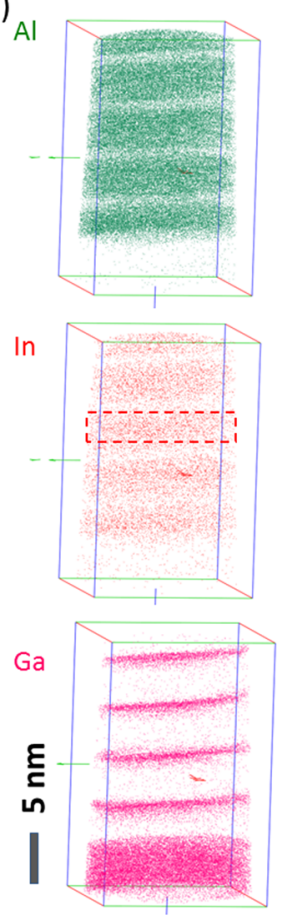

(b)

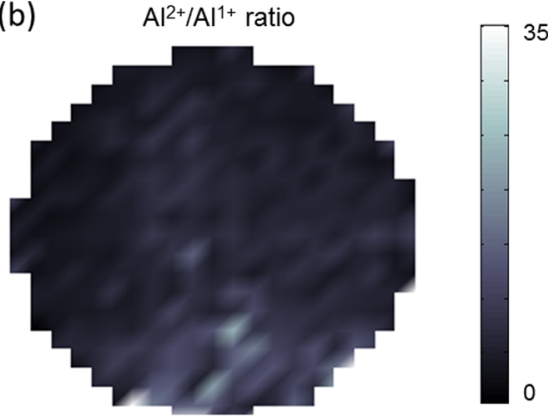

(c)

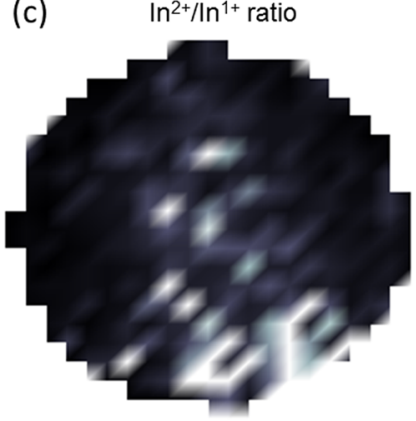

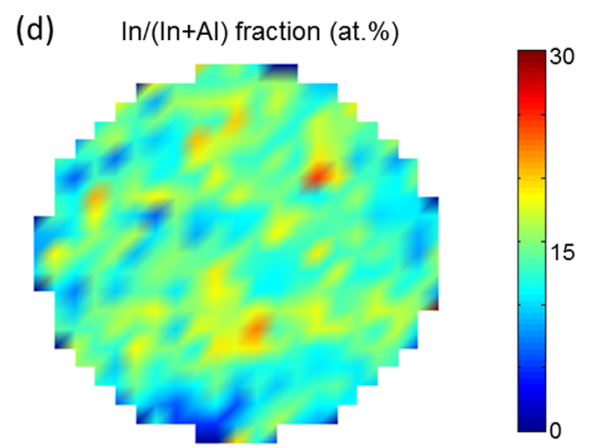

(e)
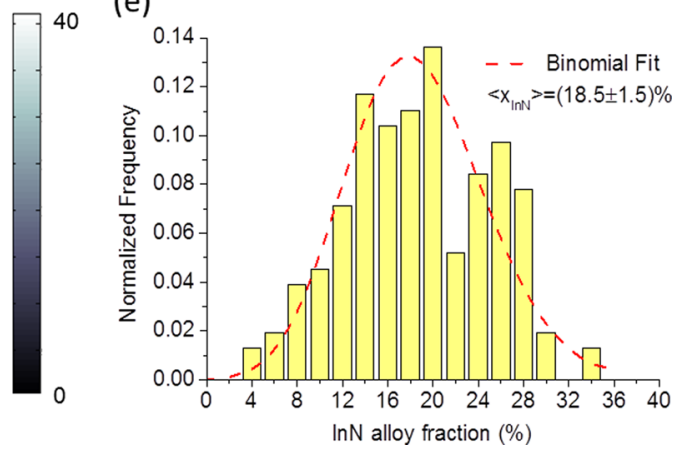

Figure 8. Study of the spatial correlation between the measured charge ratios and the $y$ InN alloy fraction in a GaN/In $\mathrm{Al}_{1-y} \mathrm{~N} / \mathrm{GaN}_{\mathrm{multiple}}$ quantum well system analyzed at $T=20 \mathrm{~K}$. (a) 3D reconstruction of the volume of the 4-fold multi-QW system: Al, In, and Ga atoms are shown from top to bottom, respectively. The red dashed rectangle indicates the analyzed subvolume. Spatial distribution of (b) the $\mathrm{Al}^{2+} / \mathrm{Al}^{1+}$ and (c) the $\mathrm{In}^{2+} / \mathrm{In}^{1+}$ charge state ratios on the detector. (d) Spatial distribution of the $y \mathrm{InN}$ fraction on the detector. (e) 3D frequency analysis of the InN fraction measured within the subvolume. The histogram is determined by sampling volume elements (voxels) containing 100 atoms each, and it is fitted with a binomial distribution (red dashed line).

Figure $7(\mathrm{~b}, \mathrm{e})$ reports the charge state ratio $\mathrm{Ga}^{2+} / \mathrm{Ga}^{1+}$ measured from the detector hitmaps averaged over the two $15 \mathrm{~nm}$ thick red boxes shown in part (a) and containing QWs \#6 and \#17, respectively. Both maps show that the field distribution is not homogeneous. A high-field region can be identified slightly left of the detector center. This high-field region can be attributed to the $m$-pole [1-100] of the wurtzite crystal. The high-field region elongates in the direction of the $a$ axis on both sides of the $m$-pole. A weaker pole is also visible close to the detector upper edge in the QW\#6 subvolume. The average $\mathrm{Ga}^{2+} / \mathrm{Ga}^{1+}$ charge state ratio is higher in the QW\#6 than in the QW\#17 hitmap. This is a consequence of the tip evolution during the analysis: due to the cone angle of $5^{\circ}$, the tip apex radius increases with the analyzed depth. The total flux being proportional to the flux per unit surface-a function of the surface field-times the square of the tip radius, a lower field is needed in order to keep the total flux constant when the tip radius increases. The progressive decrease in the average surface field is illustrated through the average $\mathrm{Ga}^{2+} / \mathrm{Ga}^{1+}$ charge state ratio for the whole data set shown in the plot of Figure $7(\mathrm{~h})$. Figures $7(\mathrm{c}, \mathrm{f})$ show the spatial distribution of the measured fraction of element III ( $\mathrm{Ga}$ and In) in the subvolumes containing QW\#6 and QW\#17, respectively. As already observed in binary GaN, the measured element III fraction is lower (higher) than 50 at. \% in high-field (low-field) regions. Furthermore, the average element III fraction progressively increases, as indicated by the plot in Figure $7(\mathrm{~h})$, which again correlates with the progressive decrease in the surface field. It is interesting to notice that both plots on Figure 7(h) exhibit an oscillation with a period of around $20 \mathrm{~nm}$ superposed to the general trend. This oscillation well correlates with the position of quantum wells. Most likely, the oscillation is determined by a local modulation of the evaporation field, which is slightly higher for the QWs than for the GaN matrix. The previous $2 \mathrm{D}$ data should be compared with the detector hitmaps of Figure $7(\mathrm{~d}, \mathrm{~g})$, relative to the $\mathrm{In} /(\mathrm{In}+\mathrm{Ga})$ fraction measured in the two subvolumes. It should be noticed that this is a reduced fraction and does not correspond to the local $\mathrm{InN}$ alloy fraction in the QW because the sampled volume is significantly larger than the QW itself. However, more detailed measurements performed with a finer sampling inside the $\mathrm{QW}$ indicate that the maximum $\mathrm{InN}$ alloy fraction in the QWs is around $20 \%{ }^{45}$ The In distribution in the two hitmaps is not uniform. Indium tends to gather in higher-concentration stripes approximately aligned with the crystal $c$-axis. It is important to underline that the In distribution is correlated neither with the field distribution nor with the element III fraction. The position of the In-rich stripes also changes from well to well, independently of the surface field and uncorrelated with the measured element III fraction. The behavior of each of the reconstructed QWs from the whole data set, is visible as a video in the Supporting Information.

3.2.2. InAIN. Figure 8 illustrates the study performed on a $\mathrm{GaN} / \mathrm{In}_{y} \mathrm{Al}_{1-y} \mathrm{~N} 5$-fold $\mathrm{QW}$ system defined on the $m$-planes of a MOCVD-grown microwire 40 . In this system, GaN corresponds to the quantum well and $\operatorname{In}_{y} \mathrm{Al}_{1-y} \mathrm{~N}$ to the barriers. The interest of this $m$-plane heterostructure is that it can be partially lattice matched to $\mathrm{GaN}$, resulting in higher material quality. For lattice-matched InAlN, the bandgap is about 4.4-4.5 eV, ${ }^{67,68}$ while the quantum wells under study exhibit PL emission at about $3.7 \mathrm{eV}^{40}$ The following data are extracted by a tip analyzed by an atom probe at $T=20 \mathrm{~K}$, illuminated by $1 \mathrm{~nJ} \mathrm{UV}$ 
laser pulses and at a flux $\Phi=0.0025-0.002$ At/pls. Part (a) of the figure shows the $3 \mathrm{D}$ reconstruction of the positions of $\mathrm{Al}$, In, and Ga atoms, respectively, as determined by APT. The red dashed rectangle indicates the subvolume extracted from one of the barriers and used for the subsequent analysis. Figure $8(b, c)$ reports the detector hitmaps of the $\mathrm{Al}^{2+} / \mathrm{Al}^{1+}$ and $\mathrm{In}^{2+} / \mathrm{In}^{1+}$ distributions, respectively. Both hitmaps are consistent and indicate that the surface field is higher in the tip region imaged in the lower part of the detector. Figure $8(\mathrm{~d})$ is the detector hitmap of the $\mathrm{In} /(\mathrm{In}+\mathrm{Al})$ fraction. As the analyzed subvolume is included in a region containing only the $\mathrm{In}_{y} \mathrm{Al}_{1-y} \mathrm{~N}$ phase, this quantity is equivalent to the $y \mathrm{InN}$ fraction in the alloy.

The hitmap indicates local fluctuations but no correlation with the surface field indicators. In order to determine if the $\mathrm{In}_{y} \mathrm{Al}_{1-y} \mathrm{~N}$ distribution is that of a random alloy, we also performed a more detailed sampling of the subvolume, in order to compare the frequency distribution of the measured $\mathrm{InN}$ alloy fraction with a binomial law. The histogram reported in Figure $8(d)$ is determined by sampling volume elements (voxels) containing 100 atoms each. It can be well fitted by a binomial law indicating that the average $\mathrm{InN}$ content is around $18 \pm 1.5 \%$, which is in fairly good agreement with the value of $15 \%$ found on other wires from the same batch by energydispersive X-ray spectroscopy (EDX) in a STEM. ${ }^{40}$

3.3. Measurement of Tb Fraction in Doped ZnO:Tb. Rare-earth doping of materials allows exploiting the optical properties of these impurity ions once introduced in a host matrix for luminescence, absorption, or light amplification purposes. In this context, we consider $\mathrm{ZnO}$ doped with $\mathrm{Tb}$ atoms ${ }^{69}$ as an interesting benchmark for testing the accuracy of composition measurement by APT. Terbium atoms are indeed introduced in the material during the growth, and a subsequent annealing stage is applied in order to have them occupy $\mathrm{Zn}$ substitutional states in the $\mathrm{Tb}^{3+}$ state, which is optically active with main photoluminescence emissions around 490 and 544 $\mathrm{nm}$. $^{43,70,71}$ The doping density obtained in the analyzed samples is around 3\% as measured by energy-dispersive X-ray spectrometry. ${ }^{43}$ (We remark that the high concentration of dopant would also make it possible to consider the material as an alloy between zinc oxide and terbium oxide. However, different stoichiometric ratios are possible for $\mathrm{Tb}_{x} \mathrm{O}_{y}$, and it is common practice to define the studied material as $\mathrm{Tb}$-doped $\mathrm{ZnO}$, briefly denoted as $\mathrm{ZnO}: \mathrm{Tb}$.)

Figure 9(a) shows a typical APT mass spectrum of $\mathrm{ZnO}: \mathrm{Tb}$, collected at $T=50 \mathrm{~K}, E_{\mathrm{las}}=0.3 \mathrm{~nJ}, V_{\mathrm{DC}}=13.1 \mathrm{kV}$, and $\Phi=2 \times$ $10^{-3} \mathrm{At} / \mathrm{pls}$. The specific contributions of $\mathrm{Tb}$-containing species can be found at $175 \mathrm{amu}\left(\mathrm{TbO}^{+}\right)$, at $87.5 \mathrm{amu}$ $\left(\mathrm{TbO}^{2+}\right)$, and at $53 \mathrm{amu}\left(\mathrm{Tb}^{3+}\right)$. (The reader should neither be confused nor infer a relationship between the charge state of the ion in the crystal matrix, conferring specific optical properties to the impurity and the charge state measured by an atom probe, which is the final result of a field evaporation process.) The other peaks are those typically found in $\mathrm{ZnO}$, as visible by direct comparison with Figure 2(c). The analysis of the field-dependent measured composition, performed according to the protocol applied for pure compounds, is reported for ensemble data in Figure 9(b). Zinc and oxygen, in the upper part of the figure, show a trend similar to that found in $\mathrm{ZnO}$, only slightly downshifted because of the presence of $\mathrm{Tb}$. The atomic fraction of $\mathrm{Tb}$, as shown in the lower part of the figure, also exhibits a weak field dependence, being between $2.4 \%$ and $2.0 \%$ at low field and stabilizing between $2.0 \%$ and $1.9 \%$ as the field increases. This trend is similar to that exhibited by $\mathrm{Zn}$.
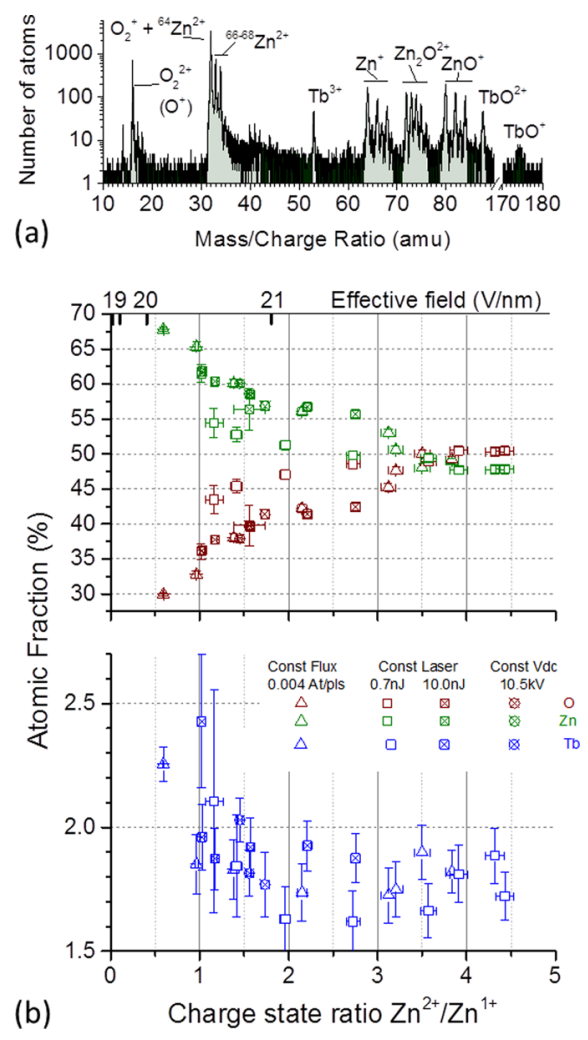

Figure 9. Influence of the surface electric field on the measured composition of $\mathrm{ZnO}: \mathrm{Tb}$. (a) Mass spectrum of a set of $\sim 10^{5}$ atoms collected from a $\mathrm{ZnO}: \mathrm{Tb}$ sample at $T=50 \mathrm{~K}, E_{\mathrm{las}}=0.3 \mathrm{~nJ}, V_{\mathrm{DC}}=13.1$ $\mathrm{kV}$, and $\Phi=2 \times 10^{-3} \mathrm{At} / \mathrm{pls}$. Terbium is field emitted either as $\mathrm{Tb}^{3+}$, or as $\mathrm{TbO}^{2+}$, or as $\mathrm{TbO}^{+}$, as indicated in the figure. The other $\mathrm{Zn}$ - and O-related species are also found in undoped $\mathrm{ZnO}$. (b) Measured fractions of $\mathrm{Zn}, \mathrm{O}$, and $\mathrm{Tb}$ as a function of the $\mathrm{Zn}^{2+} / \mathrm{Zn}^{1+}$ charge state ratio.

The field dependence of the $\mathrm{Tb}$ fraction can also be studied locally by detector hitmap analysis, as reported in Figure 10 for a data set containing $10^{6}$ atoms collected at $E_{\mathrm{las}}=0.8 \mathrm{~mW}, V_{\mathrm{dc}}$ $\sim 12.3 \mathrm{kV}$, and $\Phi=0.002 \mathrm{At} / \mathrm{pls}$ at $T=80 \mathrm{~K}$. The relationship between the locally measured $\mathrm{Zn}^{2+} / \mathrm{Zn}^{1+}$ ratios (Figure $10(\mathrm{a})$ ) and the $\mathrm{Zn}$ and $\mathrm{Tb}$ fractions (parts (b) and (c), respectively) is also accessible via the scatter plot reported in Figure 10(d). Notice that the $\mathrm{Tb}$ distribution does not exhibit any evidence of clustering, but it is not uniform: the correlation plots indicate a clear field dependence of the measured $\mathrm{Tb}$ fraction. The dashed line reported in the graph is a least-squares linear fit, whose purpose is not only that of a guide for the eyes but also indicates that a trend can be identified by statistical means.

Figure $10(d)$ reports the correlation plot in which in the $y$ axis the fraction $\mathrm{Tb} /(\mathrm{Tb}+\mathrm{Zn})$ is plotted, with an approach similar to that adopted for the quantification of the alloy fraction in III-N systems. The data show that the field dependence is thus significantly reduced.

These results point out that in the analyzed surface field regime it is difficult to establish the actual concentration of $\mathrm{Tb}$ atoms inside the $\mathrm{ZnO}$ matrix. Given the significant amount of $\mathrm{Tb}$, this also adds some uncertainty about the quantification of the total amount of $\mathrm{Zn}$ and O. Further studies specifically addressing this issue should shed more light on this point. 

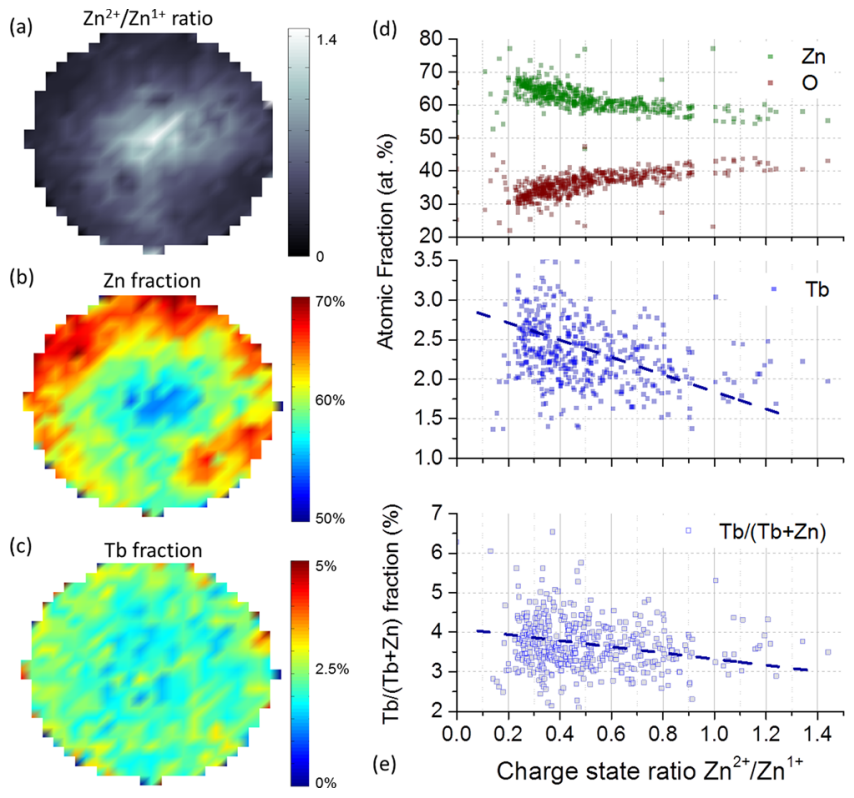

Figure 10. Study of the correlation between the surface electric field and the measured composition in $\mathrm{ZnO}: \mathrm{Tb}$. (a) Spatial distribution of the $\mathrm{Zn}^{2+} / \mathrm{Zn}^{1+}$ charge state ratio on the detector. (b) Spatial distribution of the measured $\mathrm{Zn}$ fraction and (c) of the $\mathrm{Tb}$ fraction (calculated over all atoms). (d) Correlation between the locally measured $\mathrm{Zn}^{2+} / \mathrm{Zn}^{1+}$ charge state ratio and the locally measured elemental fractions of $\mathrm{Zn}$ (green), $\mathrm{O}$ (red), and $\mathrm{Tb}$ (blue). (e) Correlation between the $\mathrm{Zn}^{2+} / \mathrm{Zn}^{1+}$ charge state ratio and the $\mathrm{Tb} /(\mathrm{Tb}$ $+\mathrm{Zn}$ ) fraction. The dashed lines in (d) and (e) are linear fits (with slopes equal to -0.011 and -0.007 , respectively) of the $\mathrm{Tb}$ data meant as a guide for the eyes, indicating a weak but significant influence of the surface field on the measured amount of $\mathrm{Tb}$. The data set consists of about $10^{6}$ atoms collected at $E_{\mathrm{las}}=0.8 \mathrm{~nJ}, V_{\mathrm{dc}} \sim 12.3 \mathrm{kV}$, and $\Phi=$ $0.002 \mathrm{At} / \mathrm{pls}$ at $T=80 \mathrm{~K}$.

\section{DISCUSSION, CONCLUSIONS, AND FUTURE OUTLOOK}

The present study of the dependence of composition measurements in selected III-N semiconductors and metal oxides allows us to draw important conclusions and formulate specific questions which should be addressed theoretically and experimentally.

For binary materials, we can identify several common elements:

- The measured composition critically depends on the experimental parameters used during the APT experiment. It can be assessed that the main parameter influencing the measurements is the electric field. This is also confirmed by the microscopic analysis of detector hitmaps, indicating a correlation between the surface field distribution and the measured composition. The field distribution on the sample surface can be related to the sample crystallography, to the specific sample shape, or to the contribution of both.

-At low field, the measurements yield an excess of the metallic species, this excess decreasing as the field is increased.

However, each material has a specific behavior, which could be summarized as follows:

-A large excess of metallic element (over 60\%) at low field is found in $\mathrm{GaN}, \mathrm{ZnO}$, and $\mathrm{MgO}$, while the composition of AlN is closer to the stoichiometric value.

- The fractions of metallic and nonmetallic elements cross in the cases of $\mathrm{GaN}, \mathrm{AlN}$, and $\mathrm{ZnO}$ but not in the case of $\mathrm{MgO}$.
-Within the same materials system (III-N or II-VI), the discrepancy between the low- and high-field composition is larger in the material with the lowest ionic character and the lowest binding energy per pair, as reported in Table 1 .

The recent studies addressing the problem of composition measurements by $\mathrm{APT}$ in $\mathrm{GaN}$ and $\mathrm{MgO}$ propose an explanation of the obtained results in these terms: (a) at low field oxygen and nitrogen may form neutral gas molecules that are evaporated without being detected; (b) at high field $\mathrm{O}$ and $\mathrm{N}$ get progressively ionized, which increases their detection efficiency, while preferential evaporation sets on for the metallic element, which decreases its detection efficiency. ${ }^{20,22-25}$ This hypothesis is consistent with the increase in background noise seen in mass spectra with increasing field and the detection efficiency estimated as 0.3 for $\mathrm{GaN}$ when the charge state ratio $\mathrm{Ga}^{2+} / \mathrm{Ga}^{1+} \sim 0.1$ allows nearly stoichiometric measurements. However, the present data cannot eliminate other possible mechanisms, such as for example molecular dissociation. To date, the only theoretical works based on first-principles calculations dedicated to this issue exclusively concerned MgO. ${ }^{53,72}$ The calculations performed by Karakha and Kreuzer, for instance, predict the evaporation of $\mathrm{O}^{+}$and suggest the formation of neutral $\mathrm{O}_{2}$ related to surface diffusion: ${ }^{53}$ it should be underlined that these calculations do not take into account the role of the laser, which is consistent with the experimentally observed behavior in terms of surface electric field only. This work also predicts a large excess of the $\mathrm{Mg}^{2+}$ charge state, consistently with our observations.

The problem of measuring a correct composition by atom probe becomes even more important when addressing the reconstruction of heterostructures, the composition of ternary alloys, and the quantification of the doping density. In the analysis of III-N ternary alloys, we showed that a correct quantification of the $x \operatorname{InN}$ alloy fraction in $\operatorname{In}_{x} \mathrm{Ga}_{1-x} \mathrm{~N}$ and $\mathrm{In}_{x} \mathrm{Al}_{1-x} \mathrm{~N}$ is possible, provided this is extracted from atom probe data as the fraction $\mathrm{In} /(\mathrm{In}+\mathrm{Ga})$ or $\mathrm{In} /(\mathrm{In}+\mathrm{Al})$, respectively. The upper limit of the alloy fraction measured in this work is about $20 \% \mathrm{InN}$. These results indicate that despite the different behavior that is found in binary III-N compounds the field ion evaporation of In inside the alloy exhibits the same behavior as that of $\mathrm{Ga}$ and $\mathrm{Al}$.

In the case of $\mathrm{ZnO}: \mathrm{Tb}$, the definition of a correct $\mathrm{Tb}$ concentration is more problematic. Ensemble data suggest that the behavior of $\mathrm{Tb}$ becomes asymptotical in the high-field limit. However, it still remains difficult to estimate whether the $\mathrm{Tb}$ atomic fraction or the $\mathrm{Tb} /(\mathrm{Tb}+\mathrm{Zn})$ fraction yields most accurate results, as in the explored field interval we could not observe any asymptotical behavior for $\mathrm{Zn}$ and $\mathrm{O}$.

For all systems studied in this work, the explanation of the compositional bias remains qualitative, hence requiring further experimental verification and a specific theoretical effort aiming to a quantitative interpretation of the different behavior of each material.

From the experimental point of view, a more extensive work is also needed, aiming at a classification of a larger quantity of materials and at the determination of the detection efficiency loss as a function of the surface field.

Furthermore, the role of the tip temperature on the evaporation behavior of the different species could be systematically addressed, in order to gain a further insight into the mechanisms leading to the loss of $\mathrm{N}$ and $\mathrm{O}$ at low field. The diffusion or migration of surface atoms may also be studied microscopically, for instance by field ion microscopy (FIM), as 
this technique has been recently applied to $\mathrm{GaN}$ and $\mathrm{MgO} .^{15,73}$ It would be indeed interesting to experimentally prove the loss of neutral species, which so far only remains a theoretical prediction.

Concerning theoretical work, the complexity of the phenomenon made it difficult to implement an atomistic approach capable of quantitative predictions of composition measurements, so far. However, the observation of specific field dependence for the present measurements could help delimit the context of the problem within a theoretical framework. DFT already provided some precious hints for the interpretation of the phenomena, ${ }^{53}$ but it cannot conclude about their dynamic aspects. Molecular dynamics could possibly yield interesting complementary insights.

In conclusion, we have defined an experimental protocol and a representation scheme based on the charge ratio of the metallic elements for testing the dependence of the measurement of composition of compound semiconductors by tomographic atom probe, and we have applied it on selected systems, namely, the binary $\mathrm{GaN}, \mathrm{AlN}, \mathrm{ZnO}$, and $\mathrm{MgO}$, and the ternary InGaN, InAlN, and $\mathrm{ZnO}: \mathrm{Tb}$. The data issued from these systems point out similar dependences of the measured compositions on the surface electric field but also important quantitative differences that still cannot be explained. A systematic application of this protocol to a larger ensemble of materials systems would certainly help improve the accuracy of atom probe measurements in compound semiconductors. The following families of materials could be profitably studied:

- Metal oxides $\left(\mathrm{Al}_{2} \mathrm{O}_{3}\right.$, iron oxides, etc.).

- Other II-VI semiconductors, such as ZnSe, CdTe, CdS, and related alloys.

- Further group III nitride alloys, such as AlGaN or high InN-containing InGaN.

- Group V nitride site alloys, such as GaAsN.

- Classic III-V binary semiconductors and alloys, such as GaAs, AlAs, and AlGaAs.

- The different SiC polytypes.

Finally, this systematic approach would allow evidencing how the evaporation behavior depends on the materials properties and pave the way to a better comprehension of field ion emission in dielectrics from both experimental and theoretical points of view.

\section{AUTHOR INFORMATION}

\section{Corresponding Author}

*E-mail: lorenzo.rigutti@univ-rouen.fr.

\section{Author Contributions}

L.M. prepared by FIB/SEM a part of the nitride samples analyzed, carried out the atom probe experiments in atom probe on all materials, analyzed the atom probe data and wrote a part of the manuscript; N.A. prepared the $\mathrm{ZnO}$ thin film samples by FIB/SEM and the nanowire samples by SEM/ $\mathrm{EBID}$, analyzed the $\mathrm{ZnO}$ and $\mathrm{ZnO}: \mathrm{Tb}$ samples by atom probe, and carried out the data analysis; D.S. prepared the $\mathrm{MgO}$ samples and performed their analysis by atom probe; I.B. prepared a part of the nitride samples by FIB/SEM; M.G. prepared by FIB/SEM and analyzed by atom probe a $\mathrm{ZnO}$ sample; W.L. analyzed the MQW sample by STEM; A.V., F.V., R.L., E.T., and P.P. contributed to planning and supervising the experiments and discussed their interpretation; X.P., A.Z., and C. Dav. grew the ZnO:Tb sample; C. Dur. and J.E. grew the InGaN/GaN microwire samples; J.F.C., R.B., and N.G. grew the GaN/InAlN microwire samples; L.R. prepared a part of the nitride samples by FIB/SEM, planned, coordinated, and supervised the atom probe experiments and their analysis, and wrote a part of the manuscript. All authors critically read and discussed the manuscript.

\section{Notes}

The authors declare no competing financial interest.

\section{ACKNOWLEDGMENTS}

We acknowledge funding through the program "Investissements d'Avenir” (ANR-10-LABX-09-01), LabEx EMC ${ }^{3}$, projects ASAP and LUZ, through the project ESp Carnot NanoTAP, through the project ANR-11-NANO-013-02 MADFIZ and through the project ANR-13-JS10-001-01 Tapoter. The authors from GPM Rouen kindly acknowledge H. J. Kreuzer and A. Bostel for fruitful discussion.

\section{REFERENCES}

(1) Gault, B.; Vurpillot, F.; Vella, A.; Gilbert, M.; Menand, A.; Blavette, D.; Deconihout, B. Design of a femtosecond laser assisted tomographic atom probe. Rev. Sci. Instrum. 2006, 77, 043705.

(2) Herbig, M.; Raabe, D.; Li, Y. J.; Choi, P.; Zaefferer, S.; Goto, S. Atomic-scale quantification of grain boundary segregation in nanocrystalline materials. Phys. Rev. Lett. 2014, 112, 126103.

(3) Kelly, T. F.; Larson, D. J.; Thompson, K.; Alvis, R. L.; Bunton, J. H.; Olson, J. D.; Gorman, B. Atom probe tomography of electronic materials. Annu. Rev. Mater. Sci. 2007, 37, 681.

(4) Perea, D.; Allen, J. A.; May, S. J.; Wessels, B. W.; Seidman, D. N.; Lauhon, L. J. Three-dimensional composition mapping of semiconductor nanowires. Nano Lett. 2006, 6, 181.

(5) Perea, D.; Hemesath, E. R.; Schwalbach, E. J.; Lensch-Falck, J. L.; Voorhees, P. W.; Lauhon, L. J. Direct measurement of dopant distribution in an individual vapour-liquid-solid nanowire. Nat. Nanotechnol. 2009, 4, 315.

(6) Chen, W. H.; Dubrovskij, V. G.; Liu, X.; Xu, T.; Lardé, R.; Nys, J. P.; Grandidier, B.; Stiévenard, D.; Patriarche, G.; Pareige, P. Boron distribution in the core of $\mathrm{Si}$ nanowire grown by chemical vapor deposition. J. Appl. Phys. 2012, 111, 094909.

(7) Moutabnabbir, O.; Isheim, D.; Blumtritt, H.; Senz, S.; Pippel, E.; Seidman, D. Colossal injection of catalyst atoms into silicon nanowires. Nature 2013, 496, 78.

(8) Da Costa, G.; Wang, H.; Duguay, S.; Bostel, A.; Blavette, D.; Deconihout, B. Advance in multi-hit detection and quantization in atom probe tomography. Rev. Sci. Instrum. 2012, 83, 123709.

(9) Koelling, S.; Richard, O.; Bender, H.; Uematsu, M.; Schulze, A.; Zschaetzsch, G.; Vandervorst, W. Direct imaging of 3D atomic-scale dopant-defect clustering processes in ion-implanted silicon. Nano Lett. 2013, 13, 2458-2462.

(10) Koelling, S.; Gilbert, M.; Goossens, J.; Hikavyy, A.; Richard, O.; Vandervorst, W. High depth resolution analysis of $\mathrm{Si} / \mathrm{SiGe}$ multilayers with the atom probe. Appl. Phys. Lett. 2009, 65, 144106.

(11) Chen, W.; Yu, L.; Misra, S.; F, Z.; Pareige, P.; Patriarche, G. Roca i Cabarrocas, P. Incorporation and redistribution of impurities into silicon nanowires during metal-particle-assisted growth. Nat. Commun. 2014, 5134.

(12) Valley, J. W.; Cavosie, A. J.; Ushikubo, T.; Reinhard, D. A.; Lawrence, D. F.; Larson, D. J.; Spicuzza, M. J. Hadean age for a post- 
magma-ocean zircon confirmed by atom-probe tomography. Nat. Geosci. 2014, 7, 219-223.

(13) Liddle, J. A.; Cerezo, A.; Grovenor, C. R. M. Position-sensitive atom probe analysis of multi quantum well structures. J. Phys. (Paris) 1989, 11, C8-437.

(14) Mazumder, B.; Vella, A.; Deconihout, B.; Al-Kassab, T. A. Evaporation mechanisms of $\mathrm{MgO}$ in laser assisted atom probe tomography. Ultramicroscopy 2011, 111, 571-575.

(15) Chen, Y.; Ohkubo, T.; Hono, K. Mechanism of laser-assisted field evaporation from insulating oxides. Ultramicroscopy 2011, 111, 562.

(16) Saxey, D. W. Correlated ion analysis and the interpretation of atom probe mass spectra. Ultramicroscopy 2011, 111, 473-479.

(17) Nishikawa, O.; Kawada, H.; Nagai, Y.; Nomura, E. Erroneous composition of GaAs mass-analyzed by atom probes. J. Phys. (Paris) 1984, 12, C9-465.

(18) Cerezo, A.; Grovenor, C. R. M. .; Smith, G. D. W. Pulsed laser atom probe analysis of III-V compound semiconductors. J. Phys. (Paris) 1986, 3, C2-309.

(19) Hashizume, T.; Hasegawa, Y.; Kobayashi, A.; Sakurai, T. Atom probe investigation of III-V semiconductors: comparison of voltage pulse and laser pulse. Rev. Sci. Instrum. 1986, 57, 1378.

(20) Agrawal, R.; Bernal, R. A.; Isheim, D.; Espinosa, H. D. Characterizing atomic composition and dopant distribution in wide band gap semiconductor nanowires using laser-assisted APT. J. Phys. Chem. C 2011, 115, 17668.

(21) Müller, M.; Smith, G. D. W.; Gault, B.; Grovenor, C. R. M. Compositional nonuniformities in pulsed laser atom probe tomography analysis of compound semiconductors. J. Appl. Phys. 2012, 111, 064908.

(22) Diercks, D. R.; Gorman, B. P.; Kirchhofer, R.; Sanford, N.; Bertness, K.; Matt Brubaker, M. Atom probe tomography evaporation behavior of $\mathrm{C}$-axis $\mathrm{GaN}$ nanowires: Crystallographic, stoichiometric, and detection efficiency aspects. J. Appl. Phys. 2013, 114, 184903.

(23) Riley, J. R.; Bernal, R. A.; Li, Q.; Espinosa, H. D.; Wang, G.; Lauhon, L. J. Atom probe tomography of a-Axis GaN nanowires: analysis of nonstoichiometric evaporation behavior. ACS Nano 2012, 6, 3898 .

(24) Riley, J. R.; Detchprohm, T.; Wetzel, C.; Lauhon, L. J. On the reliable analysis of indium mole fraction within $\operatorname{In}_{\mathrm{x}} \mathrm{Ga}_{1-\mathrm{x}} \mathrm{N}$ quantum wells using atom probe tomography. Appl. Phys. Lett. 2014, 104, 152102 .

(25) Devaraj, A.; Colby, R.; Hess, W. P.; Perea, D. E.; Thevuthasan, $S$. Role of photoexcitation and field ionization in the measurement of accurate oxide stoichiometry by laser-assisted atom probe tomography. J. Phys. Chem. Lett. 2013, 4, 933-938.

(26) Lardé, R.; Talbot, E.; Pareige, P.; Bieber, H.; Schmerber, G.; Colis, S.; Dinia, A. Evidence of superparamagnetic Co clusters in pulsed laser deposition-grown $\mathrm{Zn}_{0.9} \mathrm{Co}_{0.1} \mathrm{O}$ thin films using atom probe tomography. J. Am. Chem. Soc. 2011, 133, 1451-1438.

(27) Lardé, R.; Talbot, E.; Vurpillot, F.; Pareige, P.; Schmerber, G.; Beaurepaire, E.; Dinia, A.; Pierron-Bohnes, V. Investigation at the atomic scale of the $\mathrm{Co}$ spatial distribution in $\mathrm{Zn}(\mathrm{Co}) \mathrm{O}$ magnetic semiconductor oxide. J. Appl. Phys. 2009, 105, 126107.

(28) Mazumder, B.; Kaun, S. W.; Lu, J.; Keller, S.; Mishra, U. K.; Speck, J. S. Atom probe analysis of AlN interlayers in AlGaN/AlN/ GaN heterostructures. Appl. Phys. Lett. 2013, 111, 111603.

(29) $\mathrm{Wu}, \mathrm{J}$. When group-III nitrides go infrared: New properties and perspectives. J. Appl. Phys. 2009, 106, 011101.

(30) Stampfl, C.; Van de Walle, C. G. Density-functional calculations for III-V nitrides using the local-density approximation and the generalized gradient approximation. Phys. Rev. B 1999, 59, 5521.

(31) Zoroddu, A.; Bernardini, F.; Ruggerone, P.; Fiorentini, V. Firstprinciples prediction of structure, energetics, formation enthalpy, elastic constants, polarization, and piezoelectric constants of AlN, GaN, and InN. Phys. Rev. B 2001, 64, 045208.

(32) Xu, Y. N.; Ching, W. Y. Electronic, optical, and structural properties of some wurtzite crystals. Phys. Rev. B 1993, 48, 4335.
(33) Yu, P. Y.; Cardona, M. Fundamentals of semiconductors; SpringerVerlag: Berlin Heidelberg, 2005.

(34) Schleife, A.; Fuchs, F.; Furthmüller, J.; Bechstedt, F. Firstprinciples study of ground-and excited-state properties of $\mathrm{MgO}, \mathrm{ZnO}$, and CdO polymorphs. Phys. Rev. B 2006, 73, 245212.

(35) Rafferty, B.; Brown, L. M. Direct and indirect transitions in the region of the band gap using electron-energy-loss spectroscopy. Phys. Rev. B 1998, 58, 10326.

(36) Roessler, D. M.; Walker, W. C. Electronic spectrum and ultraviolet optical properties of crystalline MgO. Phys. Rev. 1967, 159, 733.

(37) Köster, R.; Hwang, J. S.; Durand, C.; Le Si Dang, D.; Eymery, J. Self-assembled growth of catalyst-free $\mathrm{GaN}$ wires by metal-organic vapour phase epitaxy. Nanotechnology 2010, 21, 015602.

(38) Demangeot, F.; Simeonov, D.; Dussaigne, A.; Butté, R.; Grandjean, N. Homogeneous and inhomogeneous linewidth broadening of single polar GaN/AlN quantum dots. Phys. Stat. Sol. 2009, C 6, S598.

(39) Koester, R.; Hwang, J. S.; Salomon, D.; Chen, X.; Bougerol, C.; Barnes, J. P.; Eymery, J. M-plane core-shell InGaN/GaN multiplequantum-wells on GaN wires for electroluminescent devices. Nano Lett. 2011, 11, 4839-4845.

(40) Durand, C.; Bougerol, C.; Carlin, J. F.; Rossbach, G.; Godel, F.; Eymery, J.; Grandjean, N. M-Plane GaN/InAlN multiple quantum wells in core-shell wire structure for UV emission. ACS Photonics 2014, 1, 38-46.

(41) Sallet, V.; Agouram, S.; Falyouni, F.; Marzouki, A.; Haneche, N.; Sartel, C.; Lusson, A.; Enouz-Vedrenne, S.; Muñoz-Sanjose, V.; Galtier, P. Structural characterization of one-dimensional ZnO-based nanostructures grown by MOCVD. Phys. Status Solidi B 2010, 247, $1683-1686$.

(42) Dragoe, N.; Bérardan, D.; Byl, C. On the high temperature transport properties of thermoelectric oxides. Phys. Status Solidi A 2011, 208, 140-143.

(43) Ziani, A.; Davesnne, C.; Labbé, C.; Cardin, J.; Marie, P.; Frilay, C.; Portier, X. Annealing effects on the photoluminescence of terbium doped zinc oxide films. Thin Solid Films 2014, 553, 52-57.

(44) MTI corporation, $\mathrm{MgO}$ sample description http://www.mtixtl. com/MG-a-101005S1-1.aspx, accessed September 1, 2014.

(45) Rigutti, L.; Blum, I.; Shinde, D.; Hernàndez-Maldonado, D.; Lefebvre, W.; Houard, J.; Vurpillot, F.; Vella, A.; Tchernycheva, M.; Durand, C.; et al. Correlation of microphotoluminescence spectroscopy, scanning transmission electron microscopy, and atom probe tomography on a single nano-object containing an InGaN/GaN multiquantum well system. Nano Lett. 2014, 14, 107-114.

(46) Da Costa, G.; Vurpillot, F.; Bostel, A.; Bouet, M.; Deconihout, B. Design of a delay-line position-sensitive detector with improved performance. Rev. Sci. Instrum. 2005, 76, 013304.

(47) Mathur, D.; Andersen, L. H.; Hvelplund, P.; Kella, D.; SafVan, C. P. Long-lived, doubly charged diatomic and triatomic molecular ions. J. Phys. B: At. Mol. Opt. Phys. 1995, 28, 3415.

(48) Kingham, D. R. The post-ionization of field-evaporated ions: A theoretical explanation of multiple charge states. Surf. Sci. 1982, 116, 273-301.

(49) Hagstrum, H. D.; Tate, J. T. Ionization and dissociation of diatomic molecules by electron impact. Phys. Rev. 1941, 59, 354.

(50) Larsson, M.; Baltzer, P.; Svensson, S.; Wannberg, B.; Martensson, N.; de Brito, A. N.; Karlsson, L. X-ray photoelectron, Auger electron and ion fragment spectra of $\mathrm{O}_{2}$ and potential curves of $\mathrm{O}_{2}{ }^{2+}$. J. Phys. B 1990, 23, 1175.

(51) Sugar, J.; Musgrove, A. Energy levels of zinc, Zn I through Zn XXX. J. Phys. Chem. Ref. Data 1995, 24, 1803-1872.

(52) Bachhav, M.; Danoix, F.; Hannoyer, B.; Bassat, J. M.; Danoix, R. Investigation of O-18 enriched hematite $\left(\alpha-\mathrm{Fe}_{2} \mathrm{O}_{3}\right)$ by laser assisted atom probe tomography. Int. J. Mass Spectrosc. 2013, 335, 57-60.

(53) Karahka, M.; Kreuzer, H. J. Field evaporation of oxides: A theoretical study. Ultramicroscopy 2013, 132, 54-59. 
(54) de Andrade Neto, A. V.; de Castilho, C. M. C. Some remarks on the Haydock-Kingham model for field ionization. J. Phys. B 1991, 24, 2609-2615.

(55) Zurlev, D. N.; Forbes, R. G. Field ion emission: the effect of electrostatic field energy on the prediction of evaporation field and charge state. J. Phys. D: Appl. Phys. 2003, 36, L74-L78.

(56) Lagerstedt, O.; Monemar, B. Variation of lattice parameters in GaN with stoichiometry and doping. Phys. Rev. B 1979, 19, 3064.

(57) Riley, J. R.; Padalkar, S.; Li, Q.; Koleske, D. D.; Wierer, J. J.; Wang, G. T.; Lauhon, L. J. Three-dimensional mapping of quantum wells in a $\mathrm{GaN} / \mathrm{InGaN}$ core-shell nanowire light-emitting diode array. Nano Lett. 2013, 13, 4317-4325.

(58) Galtrey, M. J.; Oliver, R. A.; Kappers, M. J.; Humphreys, C. J.; Stokes, D. J.; Clifton, P. H.; Cerezo, A. Three-dimensional atom probe studies of an $\mathrm{In}_{\mathrm{x}} \mathrm{Ga}_{1-\mathrm{x}} \mathrm{N} / \mathrm{GaN}$ multiple quantum well structures: assessment of possible indium clustering. Appl. Phys. Lett. 2007, 90, 061903.

(59) Galtrey, M. J.; Oliver, R. A.; Kappers, M. J.; McAleese, C.; Zhu, D.; Humphreys, C. J.; Clifton, P. H.; Larson, D.; Cerezo, A. Compositional inhomogeneity of a high-efficiency $\operatorname{In}_{x} \mathrm{Ga}_{1-\mathrm{x}} \mathrm{N}$ based multiple quantum well ultraviolet emitter studied by three dimensional atom probe. Appl. Phys. Lett. 2008, 92, 041904.

(60) van der Laak, N. K.; Oliver, R. A.; Kappers, M. J.; Humphreys, C. J. Characterization of InGaN quantum well with gross fluctuations in width. J. Appl. Phys. 2007, 102, 013513.

(61) van der Laak, N. K.; Oliver, R. A.; Kappers, M. J.; Humphreys, C. J. Role of gross well-width fluctuations in bright, green-emitting songle InGaN/GaN quantum well structures. Appl. Phys. Lett. 2007, 90, 121911.

(62) Bennett, S. E.; Saxey, D. W.; Kappers, M. J.; Barnard, J. S.; Humphreys, C. J. Atom probe tomography assessment of the impact of electron beam exposure on InGaN/GaN quantum wells. Appl. Phys. Lett. 2011, 99, 021906.

(63) Bennett, S. Nitride semiconductors studied by atom probe tomography and correlative techniques; Ph.D. Thesis: Cambridge, 2010.

(64) Park, C. G.; Gu, G. H.; Lee, B. H.; Jang, D. H. Effects of growth pressure on the structural and optical proerties of multi quantum wells (MQWs) in blue LED. Ultramicroscopy 2013, 127, 114.

(65) Vurpillot, F.; Gault, B.; Geiser, B.; Larson, D. J. Reconstructing atom probe data: A review. Ultramicroscopy 2013, 132, 19-30.

(66) Hono, K. Atom probe microanalysis and nanoscale microstructures in metallic materials. Acta Mater. 1999, 47, 3127-3145.

(67) Carlin, J. F.; Zellweger, C.; Dorsaz, J.; Nicolay, S.; Christmann, G.; Feltin, E.; Butté, R.; Grandjean, N. Progresses in III-nitride distributed Bragg reflectors and microcavities using AlInN/GaN materials. Phys. Status Solidi B 2005, 242, 2326.

(68) Butté, R.; Carlin, J. F.; Feltin, E.; Gonschorek, M.; Nicolay, S.; Christmann, G.; Simeonov, D.; Castiglia, A.; Dorsaz, J.; et al. Current status of AlInN layers lattice-matched to $\mathrm{GaN}$ for photonics and electronics. J. Phys. D: Appl. Phys. 2007, 40, 6238.

(69) Kouyate, D.; Ronfard-Haret, J. C.; Kossanyi, J. Photo-and electro-luminescence of rare earth-doped semiconducting zinc oxide electrodes: Emission from both the dopant and the support. J. Lumin. 1991, 50, 205-210.

(70) Liu, S. M.; Liu, F. Q.; Guo, H. Q.; Zhang, Z. H.; Wang, Z. G. Correlated structural and optical investigation of terbium-doped zinc oxide nanocrystals. Phys. Lett. A 2000, 271, 128-133.

(71) Zhang, J.; Feng, H.; Hao, W.; Wang, T. Luminescent properties of $\mathrm{ZnO}$ sol and film doped with $\mathrm{Tb}^{3+}$ ion. Mater. Sci. Eng.: A 2006, 425, 346-348.

(72) Tsukada, M.; Tamura, H.; McKenna, K. P.; Shluger, A. L.; Chen, Y. M.; Ohkubo, T.; Hono, K. Mechanism of laser assisted field evaporation from insulating oxides. Ultramicroscopy 2011, 111, 567570.

(73) Arnoldi, L.; Silaeva, E. P.; Gaillard, A.; Vurpillot, F.; Blum, I.; Rigutti, L.; Vella, A. Energy deficit of pulsed-laser field-ionized and field-emitted ions from non-metallic nano-tips. J. Appl. Phys. 2014, 115, 203705. 